\title{
ALMA captures feeding and feedback from the active galactic nucleus in NGC 613*
}

\author{
A. Audibert ${ }^{1}$, F. Combes ${ }^{1,2}$, S. García-Burillo ${ }^{3}$, L. Hunt ${ }^{4}$, A. Eckart ${ }^{5}$, S. Aalto ${ }^{6}$, V. Casasola ${ }^{7}$, F. Boone ${ }^{8}$, \\ M. Krips ${ }^{9}$, S. Viti ${ }^{10}$, S. Muller ${ }^{6}$, K. Dasyra ${ }^{11,12}$, P. van der Werf ${ }^{13}$, and S. Martín ${ }^{14,15}$ \\ 1 Observatoire de Paris, LERMA, CNRS, PSL Univ., Sorbonne University, UPMC, Paris, France \\ e-mail: anelise.audibert@obspm.fr \\ 2 Collège de France, 11 Pl. Marcelin Berthelot, 75231 Paris, France \\ 3 Observatorio Astronómico Nacional (OAN-IGN)-Observatorio de Madrid, Alfonso XII, 3, 28014 Madrid, Spain \\ ${ }^{4}$ INAF - Osservatorio Astrofisico di Arcetri, Largo E. Fermi, 5, 50125 Firenze, Italy \\ 5 I. Physikalisches Institut, Universität zu Köln, Zülpicher Str. 77, 50937 Köln, Germany \\ ${ }^{6}$ Department of Space, Earth and Environment, Chalmers University of Technology, Onsala Space Observatory, 43992 Onsala, \\ Sweden \\ 7 INAF - Isitituto di Radioastronomia, via Piero Gobetti 101, 40129 Bologna, Italy \\ 8 CNRS, IRAP, 9 Av. colonel Roche, BP 44346, 31028 Toulouse Cedex 4, France \\ 9 IRAM, 300 rue de la Piscine, Domaine Universitaire, 38406 Saint Martin d'Hères, France \\ 10 Dep. of Physics and Astronomy, UCL, Gower Place, London WC1E 6BT, UK \\ 11 Dep. of Astrophysics, Astronomy \& Mechanics, Faculty of Physics, National and Kapodistrian University of Athens, 15784 \\ Panepistimiopolis Zografou, Greece \\ 12 National Observatory of Athens, Institute for Astronomy, Astrophysics, Space Applications and Remote Sensing, Penteli 15236, \\ Athens, Greece \\ 13 Leiden Observatory, Leiden Univ., PO Box 9513, 2300 RA Leiden, The Netherlands \\ 14 European Southern Observatory, Alonso de Córdova, 3107, Vitacura, Santiago 763-0355, Chile \\ 15 Joint ALMA Observatory, Alonso de Córdova, 3107, Vitacura, Santiago 763-0355, Chile
}

Received 4 May 2019 / Accepted 1 August 2019

\begin{abstract}
We report ALMA observations of $\mathrm{CO}(3-2)$ emission in the Seyfert/nuclear starburst galaxy NGC 613, at a spatial resolution of $17 \mathrm{pc}$, as part of our NUclei of GAlaxies (NUGA) sample. Our aim is to investigate the morphology and dynamics of the gas inside the central kiloparsec, and to probe nuclear fueling and feedback phenomena. The morphology of $\mathrm{CO}(3-2)$ line emission reveals a twoarm trailing nuclear spiral at $r \lesssim 100 \mathrm{pc}$ and a circumnuclear ring at a radius of $\sim 350 \mathrm{pc}$ that is coincident with the star-forming ring seen in the optical images. Also, we find evidence for a filamentary structure connecting the ring and the nuclear spiral. The ring reveals two breaks into two winding spiral arms corresponding to the dust lanes in the optical images. The molecular gas in the galaxy disk is in a remarkably regular rotation, however the kinematics in the nuclear region are very skewed. The nuclear spectrum of $\mathrm{CO}$ and dense gas tracers $\mathrm{HCN}(4-3), \mathrm{HCO}^{+}(4-3)$, and $\mathrm{CS}(7-6)$ show broad wings up to $\pm 300 \mathrm{~km} \mathrm{~s}^{-1}$, associated with a molecular outflow emanating from the nucleus $(r \sim 25 \mathrm{pc})$. We derive a molecular outflow mass $M_{\text {out }}=2 \times 10^{6} M_{\odot}$ and a mass outflow rate of $\dot{M}_{\text {out }}=27 M_{\odot} \mathrm{yr}^{-1}$. The molecular outflow energetics exceed the values predicted by AGN feedback models: the kinetic power of the outflow corresponds to $P_{K, \text { out }}=20 \% L_{\mathrm{AGN}}$ and the momentum rate is $\dot{M}_{\text {out }} v \sim 400 L_{\mathrm{AGN}} / c$. The outflow is mainly boosted by the AGN through entrainment by the radio jet, but given the weak nuclear activity of NGC 613, we might be witnessing a fossil outflow resulting from a previously strong AGN that has now faded. Furthermore, the nuclear trailing spiral observed in CO emission is inside the inner Lindblad resonance ring of the bar. We compute the gravitational torques exerted in the gas to estimate the efficiency of the angular momentum exchange. The gravity torques are negative from 25 to $100 \mathrm{pc}$ and the gas loses its angular momentum in a rotation period, providing evidence for a highly efficient inflow towards the center. This phenomenon shows that the massive central black hole has significant dynamical influence on the gas, triggering the inflowing of molecular gas to feed the black hole.
\end{abstract}

Key words. galaxies: active - galaxies: kinematics and dynamics - submillimeter: ISM - ISM: jets and outflows galaxies: evolution - galaxies: individual: NGC 613

\section{Introduction}

The energy of active galactic nuclei (AGN) is well understood as being due to gas accretion onto the supermassive black hole (SMBH; Antonucci 1993). Gas inflows into the center of galaxies can fuel the SMBH and the energy input by the AGN can trig-

\footnotetext{
* Reduced datacubes and images are only available at the CDS via anonymous ftp to cdsarc.u-strasbg.fr (130.79.128.5) or via http://cdsarc.u-strasbg.fr/viz-bin/cat/J/A+A/632/A33
}

ger subsequent feedback. The feedback can in turn regulate the SMBH growth and suppress star formation (e.g., Croton et al. 2006; Sijacki et al. 2007). Feeding and feedback are key processes in the co-evolution of black holes (BH) and their host galaxies, which is now well established by the tight $\mathrm{M}-\sigma$ relation (e.g., Magorrian et al. 1998; Gültekin et al. 2009; McConnell \& Ma 2013). It is important to study the efficiency of angular momentum transport in galaxy disks in order to understand how the star formation and nuclear activity are fueled and to elucidate 
the timescales involved, since both feeding processes rely on a common cold gas supply, but in different periods of time $\left(\sim 10^{5} \mathrm{yr}\right.$ for $\mathrm{BH}$ growth and $\sim 10^{7-9} \mathrm{yr}$ for star formation, GarcíaBurillo et al. 2016). These timescales are related to the mechanisms that drive the gas from galactic scales $(\sim 10 \mathrm{kpc})$ to nuclear scales (a few pc), through removal of angular momentum; large nonaxisymmetric perturbations, such as bars or spirals, represent one of these mechanisms, but so far no unique physical process associated with inward transport of gas in galaxy disks has been identified.

On large scales, cosmological simulations show that mergers and galaxy interactions are able to produce strong nonaxisymmetries (e.g., Hopkins et al. 2006; Di Matteo et al. 2008). On kiloparsec scales, bar instabilities, either internally driven by secular evolution or triggered by companions, can first feed a central starburst and then fuel the BH (García-Burillo et al. 2005). On the other hand, gas inflow is impeded by the inner Lindblad resonance (ILR), where the gas is trapped in a nuclear ring (see Piner et al. 1995; Regan \& Teuben 2004). On scales of a few hundred parsecs, the "bars within bars" scenario (e.g., Shlosman et al. 1989), together with $m=1$ instabilities and nuclear warps (Schinnerer et al. 2000), takes over as a dynamical mechanism (see e.g., Hunt et al. 2008). Observations of nearby low-luminosity AGN (LLAGN) with the NUGA program have revealed smoking-gun evidence of AGN fueling in one third of galaxies (García-Burillo \& Combes 2012). This result suggests that galaxies alternate periods of fueling and starvation, and might be found in a feeding phase at $300 \mathrm{pc}$ scales only one third of the time.

As we approach the center of galaxies, other mechanisms can contribute to the fueling: viscous torques, from dense gas in regions of large shear, or dynamical friction can drive massive clouds to the nucleus (e.g., Combes 2003; Jogee et al. 2006). Simulations suggest that fueling involves a series of dynamical instabilities $(m=2, m=1)$ on scales of $\sim 10 \mathrm{pc}$, and also predict the formation of a thick gas disk similar to the putative torus invoked to explain obscured AGN (Hopkins \& Quataert 2010; Hopkins et al. 2012). These fueling episodes are eventually quenched by either nuclear star formation winds or AGN feedback.

The recent discovery of many massive (a few $10^{7} M_{\odot}$ ) molecular outflows in nearby AGN (e.g., Fischer et al. 2010; Feruglio et al. 2010, 2017; Alatalo et al. 2011; Sturm et al. 2011; Veilleux et al. 2013; Cicone et al. 2014; Sakamoto et al. 2014; García-Burillo et al. 2014; Dasyra \& Combes 2012; Dasyra et al. 2014) has promoted the idea that winds may be major actors in sweeping gas out of galaxies. It has already been established that mass-outflow rates increase with AGN luminosity, supporting the idea of a luminous AGN pushing away the surrounding gas through a fast wind. Observational works (Cicone et al. 2014; Fiore et al. 2017; Fluetsch et al. 2019) have shown that molecular outflow properties are correlated with AGN luminosity, where the outflow kinetic power corresponds to about $5 \% L_{\mathrm{AGN}}$ and the momentum rate is $\sim 20 L_{\mathrm{AGN}} / c$, in agreement with theoretical models of AGN feedback (Faucher-Giguère \& Quataert 2012; Zubovas \& King 2012, 2014). Outflows have been traced for a long time in ionized or atomic gas (Rupke et al. 2005a; Riffel \& Storchi-Bergmann 2011), making it now possible to compare the different gas phases. Carniani et al. (2015) found that ionized gas only traces a small fraction of the total gas mass, suggesting that the molecular phase dominates the outflow mass. This trend is also found by Fiore et al. (2017), but the ratio between molecular and ionized mass-outflow rates is reduced at the highest AGN bolometric luminosities.
Probing AGN feeding and feedback phenomena through the kinematics and morphology of the gas inside the central kiloparsec has only recently been possible due to the unprecedented spatial resolution and sensitivity of the Atacama Large Millimeter/submillimeter Array (ALMA). Evidence of AGN feeding was found in NGC 1566, where a molecular trailing spiral structure from 50 to $300 \mathrm{pc}$ was detected with ALMA Cycle 0 observations, and according to its negative gravity torques, is found to be contributing to the fueling of the central $\mathrm{BH}$ (Combes et al. 2014). Again using Cycle 0 observations, a molecular outflow is also seen in the LLAGN in the Seyfert 2 NGC 1433. It is the least massive molecular outflow $\left(\sim 4 \times 10^{6} M_{\odot}\right)$ ever detected around galaxy nuclei (Combes et al. 2013). Furthermore, a fast and collimated outflow has been detected in $\mathrm{HCN}(1-0)$ and $\mathrm{CO}(1-0)$ emission in the nucleus of Arp 220, extending up to $120 \mathrm{pc}$ and reaching velocities up to $\pm 840 \mathrm{~km} \mathrm{~s}^{-1}$ (BarcosMuñoz et al. 2018).

In the prototypical Seyfert 2 NGC 1068, a clear molecular ouflow has also been detected, entrained by the AGN radio jets (Krips et al. 2011; García-Burillo et al. 2014). NGC 1068 is also the first case where the molecular torus is resolved, using the continuum and the $\mathrm{CO}(6-5), \mathrm{HCN}(3-2)$, and $\mathrm{HCO}^{+}(3-2)$ emission lines observed with ALMA (Gallimore et al. 2016; García-Burillo et al. 2016; Imanishi et al. 2016). The dynamics of the molecular gas in the NGC 1068 torus revealed strong noncircular motions and enhanced turbulence superposed on a slow rotation pattern of the disk. The AGN is clearly off-center with respect to the torus, implying an $m=1$ perturbation (García-Burillo et al. 2016). Recently, we reported observations of molecular tori around massive BHs in a sample of seven nearby LLAGN (Seyfert/ Low Ionization Nuclear Emission-line Regions- LINERs), at the unprecedented spatial resolution of 3-10 pc (Combes et al. 2019, hereafter Paper I). The ALMA observations bring a wealth of new information on the decoupled molecular tori, which are found to have radii ranging from 6 to $27 \mathrm{pc}$, to be unaligned with the orientation of the host galaxy, and to frequently be slightly off-center with regards to the AGN position. The kinematics of the gas inside the sphere of influence (SoI) of the central $\mathrm{BH}$ also allowed us to estimate the $\mathrm{BH}$ masses $\left(M_{\mathrm{BH}} \sim 10^{7-8} M_{\odot}\right)$.

In this paper, we present the combined ALMA cycle 3 and 4 observations in the $\mathrm{CO}(3-2)$ line of the Seyfert galaxy NGC 613, with a spatial resolution of $17 \mathrm{pc}$. These observations were part of the sample presented in Paper I, but the data analyzed here have a higher sensitivity, allowing faint broad wings to be detected that are usually associated to outflows. This object presents a nuclear trailing spiral and we discovered a molecular outflow in its nuclear region. Therefore, NGC 613 is a special case allowing us to study the complexity of fuelling and feedback mechanisms in AGN, and to perform a detailed analysis of the gas flow cycle in AGN. All relevant characteristics of NGC 613 are described below. Observations are detailed in Sect. 2 and results are presented in Sect. 3. The properties of the nuclear molecular outflow discovered in the very center of NGC 613 are discussed in Sect. 4. The interpretation of inflowing gas in terms of gravitational torques is discussed in Sect. 5, and conclusions are drawn in Sect. 6.

NGC 613 is a nearby barred SB(rs)bc galaxy (de Vaucouleurs et al. 1991) at a distance of $17.2 \mathrm{Mpc}\left(1^{\prime \prime}=83 \mathrm{pc}\right)$. It has a largescale bar of $r_{\text {bar }} \sim 90^{\prime \prime}$ with a position angle (PA) of $127^{\circ}$ and a secondary nuclear bar with $\mathrm{PA}=122^{\circ}$ (Jungwiert et al. 1997; Seigar et al. 2018). Judging from NED, NGC 613 is only moderately inclined, with inclination $\sim 38^{\circ}$ (see also Sect. 3.4). Prominent dust lanes are visible along the large-scale bar and the 

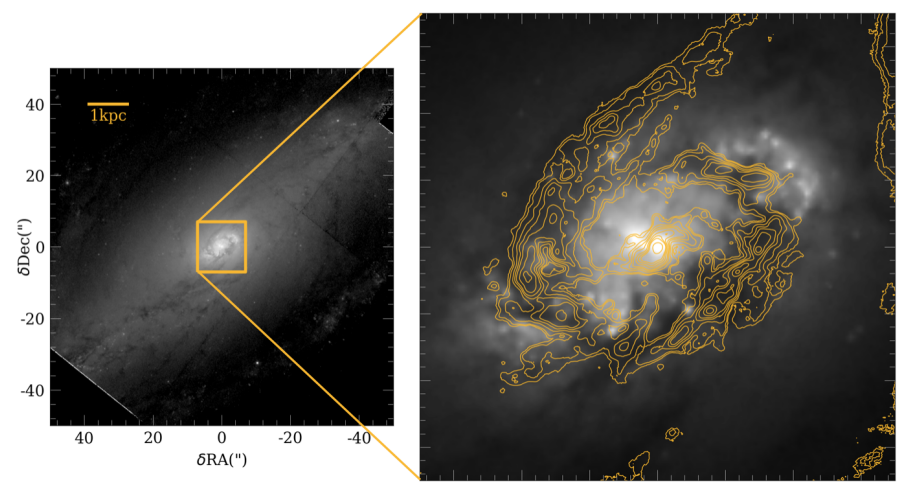

Fig. 1. WFC/F814W HST image in the left panel, showing the nuclear ring and the sets of spiral arm structures at large scales. The scale is indicated in the upper-left corner. Right: $14^{\prime \prime}$ square zoom showing the $\mathrm{CO}(3-2)$ contours overlaid on the same image. The HST image has been aligned to match the ALMA astrometry.

Table 1. Properties of NGC 613.

\begin{tabular}{lcc}
\hline \hline Parameter & Value & Reference \\
\hline$\alpha_{J 2000}{ }^{(a)}$ & $01 \mathrm{~h} 34 \mathrm{~m} 18.23 \mathrm{~s}$ & $(1)$ \\
$\delta_{J 2000}{ }^{(a)}$ & $-29 \mathrm{~d} 25 \mathrm{~m} 06.56 \mathrm{~s}$ & $(1)$ \\
$V_{\text {hel }}$ & $1481 \pm 5 \mathrm{~km} \mathrm{~s}^{-1}$ & $(1)$ \\
RC3 Type & $\mathrm{SB}(\mathrm{rs}) \mathrm{bc}$ & $(1)$ \\
Nuclear activity & $\mathrm{Sy} / \mathrm{Starburst}$ & $(1,2)$ \\
Inclination & $41^{\circ}$ & $(3)$ \\
Position angle $^{\circ}$ & $120^{\circ}$ & $(3)$ \\
Distance $^{(b)}$ & $17.2 \mathrm{Mpc}^{(c)}$ & $(1)$ \\
SFR $^{(c)}$ & $5.3 M_{\odot} \mathrm{yr}^{-1}$ & $(1)$ \\
$M_{\mathrm{HI}}$ & $4.7 \times 10^{9} M_{\odot}$ & $(4)$ \\
$M_{*}-$ Stellar mass & $4.5 \times 10^{10} M_{\odot}$ & $(5)$ \\
$L_{\mathrm{IR}}{ }^{(d)}$ & $3 \times 10^{10} L_{\odot}$ & $(6)$ \\
$\alpha_{J 2000}{ }^{(d)}$ & $01 \mathrm{~h} 34 \mathrm{~m} 18.19 \mathrm{~s}$ & $(7)$ \\
$\delta_{J 2000}{ }$ & $-29 \mathrm{~d} 25 \mathrm{~m} 06.59 \mathrm{~s}$ & $(7)$ \\
\hline
\end{tabular}

Notes. ${ }^{(a)}\left(\alpha_{J 2000}, \delta_{J 2000}\right)$ is the phase tracking center of our interferometric observations. ${ }^{(b)}$ Distances are the median values of $\mathrm{z}$-independent distances from NED Steer et al. (2017). ${ }^{(c)}$ Star formation rate (SFR) is derived from infrared luminosities (NED). ${ }^{(d)}$ The RA-Dec positions are the new adopted center, derived from the central continuum peak in this work, with an uncertainty of $\sim 0.1^{\prime \prime}$ (see Sect. 3.1).

References. (1) NASA/IPAC Extragalactic Database (NED); (2) VeronCetty \& Veron (1986); (3) (de Vaucouleurs et al. 1991); (4) Gadotti et al. (2019); (5) Combes et al. (2019); (6) (Sturm et al. 2002); (7) this work.

presence of multiple spirals arms gives NGC 613 a "tentacular" appearance. NGC 613 has a typical inner Lindblad resonance (ILR) nuclear ring of $\sim 3.5^{\prime \prime}(300 \mathrm{pc})$ in radius just inside the two characteristic leading dust lanes of the bar (Fig. 1).

This galaxy hosts a LLAGN, that was first classified as a composite Seyfert/HII object by Veron-Cetty \& Veron (1986) based on its low-resolution optical spectrum, and later confirmed through MIR spectroscopy (Goulding \& Alexander 2009) and $\mathrm{X}$-ray observations using the ROSAT and XMM-Newton (Liu \& Bregman 2005; Castangia et al. 2013, respectively). Water masers have been detected in the nucleus by Kondratko et al. (2006).

NGC 613 shows clear evidence of star formation, shock excitation, and AGN activity (Davies et al. 2017). Radio continuum observations show evidence for a collimated jet from the $\mathrm{AGN}$ and a nuclear ring with a moderate inclination of $i \sim 55^{\circ}$
(Hummel et al. 1987; Hummel \& Jorsater 1992). The presence of the outflow has already been suggested by the high-velocity dispersion of the [FeII] line along the radio jet (Falcón-Barroso et al. 2014). The ring-like structure in Br $\gamma$ emission in NGC 613, comprising "hot spots" of current massive star formation (Böker et al. 2008; Falcón-Barroso et al. 2014), indicates an ongoing starforming episode. We collect the main properties of NGC 613 in Table 1.

\section{Observations}

We report the combined ALMA Cycle 3 and Cycle 4 observations of $\mathrm{CO}(3-2), \mathrm{CS}(7-6), \mathrm{HCN}(4-3)$, and $\mathrm{HCO}^{+}(4-3)$ and continuum in band 7 , at rest frame frequencies $v_{\text {rest }}$ of 345.8 , $342.9,354.5,356.7$, and $350 \mathrm{GHz}$, respectively. The observations of the NUGA sample are described in Paper I; here we include additional details for NGC 613.

In Cycle 3, NGC 613 was observed (project ID: \#2015.1.00 404.S, PI F. Combes) simultaneously in $\mathrm{CO}(3-2), \mathrm{HCO}^{+}(4-3)$, and $\mathrm{HCN}(4-3)$ for both the compact (TC, baselines 15 to $630 \mathrm{~m}$ ) and the extended (TE, baselines 15 to $1400 \mathrm{~m}$ ) configurations. The largest recoverable angular scale corresponding to the shortest baseline is about $12^{\prime \prime}$. The TC configuration was observed in April 2016 with 40 antennas and an integration time, including calibration and overheads, of $20 \mathrm{~min}$, providing a synthesized beam of $\sim 00^{\prime \prime} 38$. The TE configuration was observed in August 2016 with 41 antennas, total integration of $40 \mathrm{~min}$ and a synthesized beam of $\sim 00^{\prime \prime} 14$. The correlator setup, designed to simultaneously observe three lines, provided a velocity range of $1600 \mathrm{~km} \mathrm{~s}^{-1}$ for each line, but did not center the $\mathrm{HCO}^{+}(4-3)$ and $\mathrm{HCN}(4-3)$ lines $\left(200 \mathrm{~km} \mathrm{~s}^{-1}\right.$ on one side and $1400 \mathrm{~km} \mathrm{~s}^{-1}$ on the other, which is adequate for a nearly face-on galaxy), and $1800 \mathrm{MHz}$ bandwidth in the continuum.

The Cycle 4 observations were carried out in November 2016 and July 2017 (project ID: \#2016.1.00296.S, PI F. Combes) at higher spatial resolution $(\sim 6.5 \mathrm{pc})$ aiming at resolving the molecular torus. The tuning configuration of Band 7 was in the $\mathrm{CO}(3-2)$ and $\mathrm{HCO}^{+}(4-3)$ lines and the continuum to avoid a restricted velocity range in the expected broader spectral lines towards the nucleus. The correlator setup was selected to center the $\mathrm{CO}(3-2)$ and the $\mathrm{HCO}^{+}$lines in the $2 \mathrm{GHz}$ bandwidth. The compact configuration (TM2, baselines 19 to $500 \mathrm{~m}$ ) was observed with 44 antennas for an integration time of 14 min and a synthesised beam of 0 '.31 and the extended (TM1, baselines 19 to $3100 \mathrm{~m}$ ) was observed with 43 antennas for $1.2 \mathrm{~h}$ and a synthesised beam of $\sim 0$ '” 08 .

The phase center of the observations was that of the nucleus (Table 1), with a single pointing covering a field of view (FoV) of $18^{\prime \prime}$. The galaxy was observed in dual polarization mode with $1.875 \mathrm{GHz}$ total bandwidth per spectral window, and a channel spacing of $0.488 \mathrm{MHz}$ corresponding to $\sim 0.8 \mathrm{~km} \mathrm{~s}^{-1}$, after Hanning smoothing. The flux calibration was done with radio quasars close to the position of the target in the sky, which are regularly monitored at ALMA, and resulted in 10\% accuracy.

The data from Cycles 3 and 4 were calibrated and concatenated with the CASA software (version from 4.5.3 to 4.7.2, (McMullin et al. 2007), and the imaging and cleaning were performed with the GILDAS software (Guilloteau \& Lucas 2000). In Paper I, we used only the most extended configurations (TM1+TE), but in this work we have combined all the configurations to improve the sensitivity. The analysis was performed in GILDAS together with PYTHON packages (radio-astro-tools, APLpy, PySpecKit Ginsburg et al. 2015; Robitaille \& Bressert 2012; Ginsburg \& Mirocha 2011). The CLEANing was performed 
using the Hogbom method and a natural weighting in order to achieve the best sensitivity, resulting in a synthesised beam of $0 \prime \prime 21 \times 0, \prime 19$ for the concatenated data cube. The spectral line maps were obtained after subtraction of the continuum in the $u v$-plane using the tasks UV_CONTINUUM and UV_SUBTRACT. The data cubes were then smoothed to $10 \mathrm{~km} \mathrm{~s}^{-1}(11.5 \mathrm{MHz})$. The total integration time provided an $\mathrm{rms}$ of $87 \mu \mathrm{Jy} \mathrm{beam}^{-1}$ in the continuum, and $0.43 \mathrm{mJy}^{-1} \mathrm{bem}^{-1}$ in the line channel maps per channel of $10 \mathrm{~km} \mathrm{~s}^{-1}$ (corresponding to $\sim 1 \mathrm{~K}$, at the obtained spatial resolution). The final maps were corrected for primary beam attenuation. Very little $\mathrm{CO}(3-2)$ emission was detected outside the full width at half power (FWHP) primary beam.

Due to a lack of very short baselines $(<15 \mathrm{~m})$, extended emission was filtered out at scales larger than $12^{\prime \prime}$ in each channel map. Since the velocity gradients are high in galaxy nuclei, the line measurements are not significantly affected; indeed the size in each velocity channel is not expected to be extended.

\section{Results}

\subsection{Continuum emission}

Previous ALMA band 3 and 7 observations by Miyamoto et al. (2017) detect continuum emission from both the circumnuclear disk (CND) and the star-forming ring $(250<r<340 \mathrm{pc})$. At $95 \mathrm{GHz}$ with a $\sim 0$ '! 6 resolution, these latter authors found a continuum jet with $\mathrm{PA}=20^{\circ}$, which corresponds to the $4.9 \mathrm{GHz}$ and 14.9 GHz jets (Hummel \& Jorsater 1992), close to the minor axis of the ring. In the nucleus, the negative spectral index, $\alpha \sim-0.6$, is compatible with synchrotron emission, with a small fraction of free-free emission, while the index $\alpha \sim-0.2$ along the starforming ring could be from free-free emission (Miyamoto et al. 2017).

At our resolution of 0 !'2 $(\sim 17 \mathrm{pc})$, the central continuum is resolved at $350 \mathrm{GHz}$, with some compact emission along the star-forming ring, as display in Fig. 2. The $\sim 2.2 \mathrm{mJy}$ peak emission is detected at $25 \sigma$ significance. We determine the peak of the continuum emission by fitting a circular Gaussian source in the $u v$-plane using the GILDAS UV_FIT task. The fitted results in the central continuum emission for the flux is $2.4 \pm 0.1 \mathrm{mJy}$ and for the RA and Dec relative to the phase center are $\triangle \mathrm{RA}=$ $-0^{\prime \prime} .587$ and $\Delta \mathrm{Dec}=-0^{\prime \prime} .03219$, with a relative uncertainty of $\pm 00^{\prime \prime} 003$. These values are listed as references of the new adopted center and AGN position in Table 1. Within the error bar, the AGN position is consistent with the positions derived by Miyamoto et al. (2017) and from X-ray observations (Liu \& Bregman 2005).

\subsection{Molecular gas distribution and morphology}

Figure 3 displays the $\mathrm{CO}(3-2)$ channel maps, with a velocity range of $450 \mathrm{~km} \mathrm{~s}^{-1}$ and a velocity resolution of $10 \mathrm{~km} \mathrm{~s}^{-1}$. The channels show evidence for a regular velocity field in a ring at a radius $\sim 3.5^{\prime \prime}(300 \mathrm{pc}$ ), with two winding-arm structures coming from the NW and SE directions. These spiral arms coincide with the beginning of the dust lanes along the bar seen in the HST/F814W image (Fig. 1); they are the contact points between the tangent dust lanes and the ring.

At small radii, there is a spiral structure in the central channels $\left( \pm 100 \mathrm{~km} \mathrm{~s}^{-1}\right)$ that can be more clearly seen in Fig. 4. The two-arm nuclear gas spiral at $r \lesssim 100 \mathrm{pc}$ is trailing toward the center; this is discussed further in Sect. 5. Additionally, the velocity distribution is perturbed for channels in the range \pm 50 $100 \mathrm{~km} \mathrm{~s}^{-1}$ for $r \sim 150 \mathrm{pc}$, and the morphology shows evidence

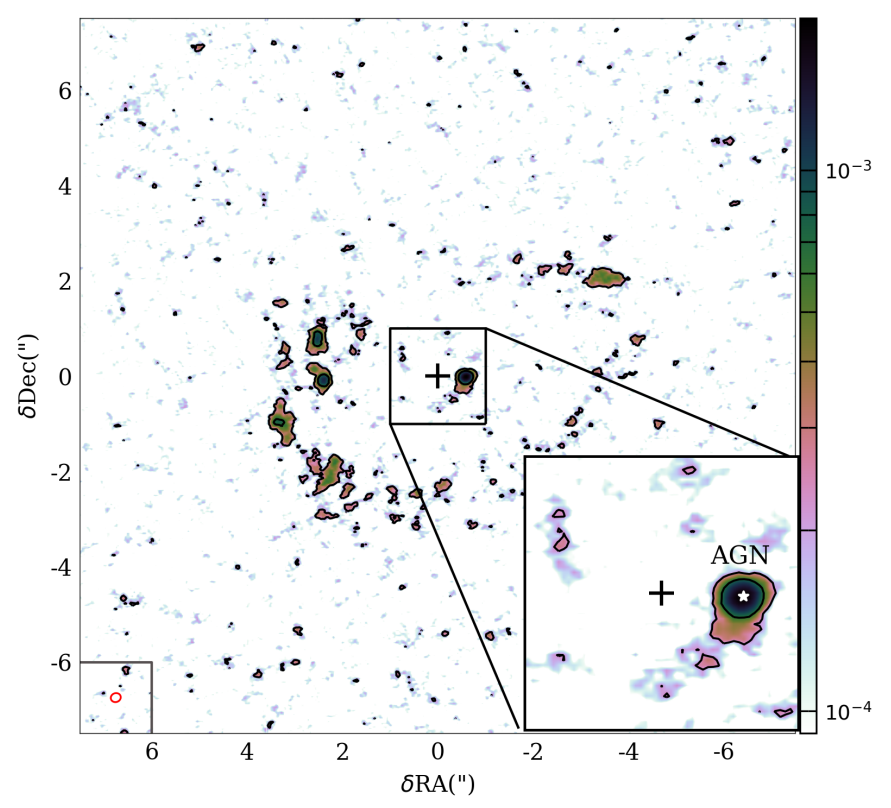

Fig. 2. Continuum emission at $0.87 \mathrm{~mm}$. The central $15^{\prime \prime} \times 15^{\prime \prime}$ are shown in the main panel, the zoom-in of the central $2^{\prime \prime} \times 2^{\prime \prime}$ is shown in the bottom right corner. The cross indicates the phase center, while the white star indicates the new center adopted in Table 1. The color scale is in Jy/beam. The beam size $\left(0.21^{\prime \prime} \times 0.19^{\prime \prime}\right)$ is shown in the red ellipse in the bottom left corner.

for a filamentary structure connecting the ring and the nuclear spiral.

We constructed the moment maps of the $\mathrm{CO}(3-2)$ line, clipping the emission at $<5 \sigma_{\text {rms. }}$. The integrated intensity (zeromoment) map in the left panel of Fig. 4 shows that the $\mathrm{CO}$ emission follows the $\sim 300 \mathrm{pc}$ star-forming circumnuclear ring. Miyamoto et al. (2017) mapped the ring in $\mathrm{CO}(1-0)$ and $\mathrm{CO}(3-2)$ with ALMA at 0..7 and 0,.4, respectively, and found a clumpy ring that is globally regular but has spots of active and efficient star formation. In our maps at higher resolution, we find that the molecular emission in the ring is clumpy and incomplete, and coincides with the same star forming clumps observed in $\mathrm{Br} \gamma$ in the near-infrared (NIR; see Falcón-Barroso et al. 2014, and Sect. 3.6). The $\mathrm{CO}(3-2)$ emission peak of $25 \mathrm{Jy} \mathrm{km} \mathrm{s}^{-1}$ beam $^{-1}$ corresponds to the AGN position reported in Table 1. Within the CND, there is a clear trailing two-arm spiral structure. The ring reveals two breaks into two winding spiral arms, at NW and SE. The main morphological features are shown in the sketch of the galaxy in Fig. 5.

We superposed the $\mathrm{CO}(3-2)$ contours onto the HST maps in the F814W filter ${ }^{1}$ shown in Fig. 1. This reveals a remarkable similarity in morphology: the molecular ring seen in the $\mathrm{CO}$ emission coincides with the dusty nuclear ring in the HST image, and the winding arms are the beginning of the characteristic dust lanes along the bar. At the very center $(\lesssim 100 \mathrm{pc})$ however, the stellar and molecular morphologies are dissimilar.

In the middle panel of Fig. 4, the intensity-weighted velocity (first-moment) map shows a clear rotation pattern in the galaxy plane, with velocities peaking in the range $\sim \pm 200 \mathrm{~km} \mathrm{~s}^{-1}$ from the systemic velocity $\left(v_{\text {sys }} 1481 \mathrm{~km} \mathrm{~s}^{-1}\right.$; see discussion below). The velocity distribution and morphology in the central $200 \mathrm{pc}$ are more perturbed due to the filamentary streams and the

1 The HST image was aligned to the ALMA astrometry, and the peak emission in the HST image was recentered to the AGN position in Table 1. 


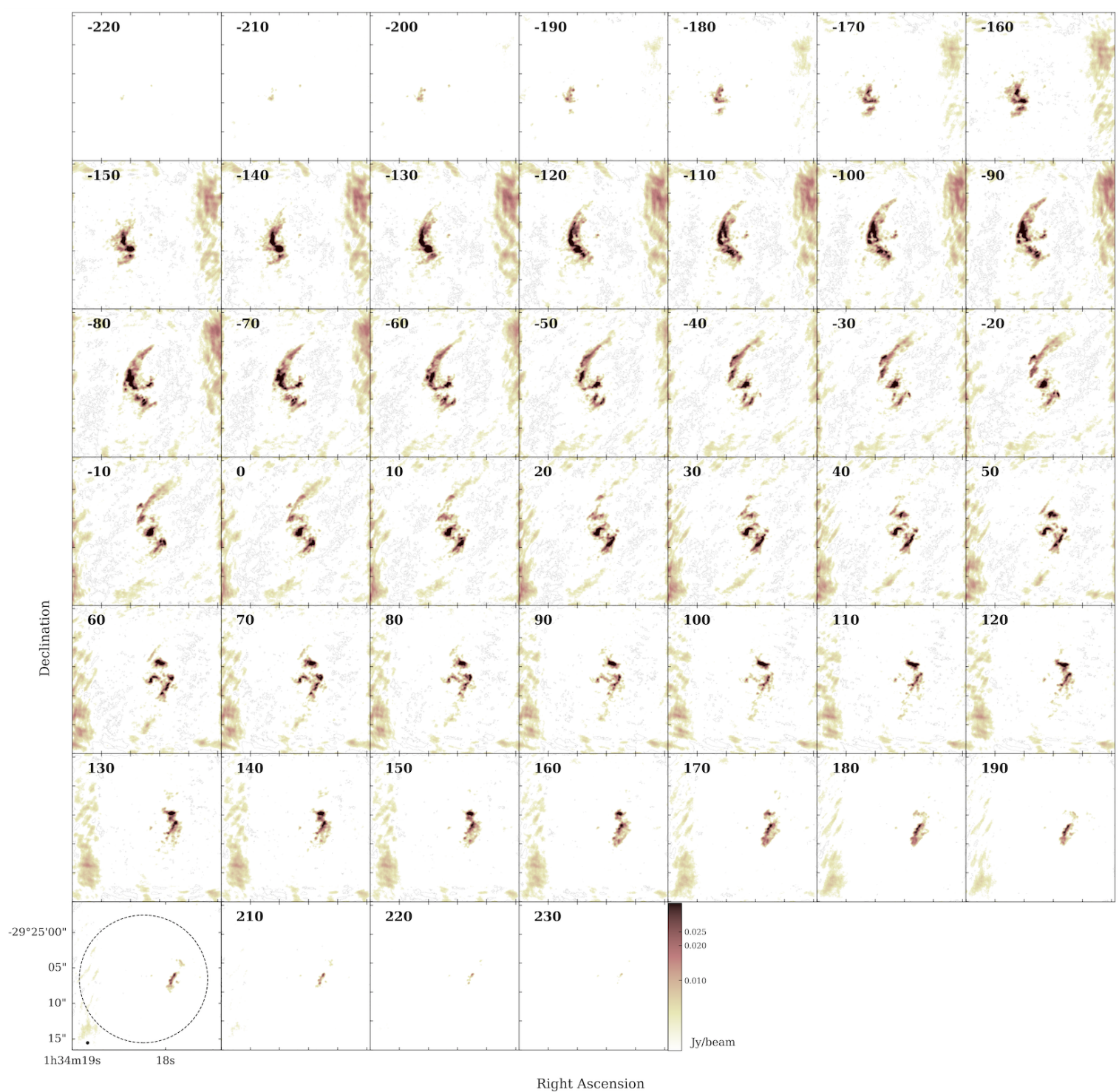

Fig. 3. Channel maps of $\mathrm{CO}(3-2)$ emission in the center of NGC 613 using the combined observations from ALMA Cycles 3 and 4. We present the channel maps from -220 (top left) to $+230 \mathrm{~km} \mathrm{~s}^{-1}$ (bottom right) relative to the $v_{\text {sys }}=1471 \mathrm{~km} \mathrm{~s}^{-1}$, in steps of $10 \mathrm{~km} \mathrm{~s}^{-1}$. Each of the 46 square boxes is $21^{\prime \prime} \times 21^{\prime \prime}$ in size, while the primary beam is $18^{\prime \prime}$ in diameter and is indicated in the dashed circle on the bottom left panel. The synthesized beam $\left(0^{\prime \prime}: 21 \times 0^{\prime \prime} \cdot 19, \mathrm{PA}=-69^{\circ}\right)$ is shown in the black ellipse in the bottom left corner. The center of the maps is the phase center of the interferometric observations given in Table 1 . The color scale is in power stretch (with a power index of 0.5 ) ranging between 2 and $38 \mathrm{mJy}^{\mathrm{b}}$ beam ${ }^{-1}$.

nuclear spiral. The NW winding arm is mostly blueshifted and the SE redshifted, indicating that rotation and possibly gas pileup are taking place; this is discussed further in Sect. 5.

Close to the AGN, the velocity dispersion is high $(\sigma \sim$ $130 \mathrm{~km} \mathrm{~s}^{-1}$ ), as displayed in the right panel of Fig. 4 (secondmoment map). In the nuclear spiral the velocity dispersion ranges from $70 \sim 120 \mathrm{~km} \mathrm{~s}^{-1}$ and the average dispersion along the ring is $\sim 40 \mathrm{~km} \mathrm{~s}^{-1}$, with more elevated values in the clumpy regions. Furthermore, we can distinguish a disturbance in the overdense region in the west part of the ring, with an increased dispersion of $\gtrsim 150 \mathrm{~km} \mathrm{~s}^{-1}$. This region also corresponds to an enhanced spot observed in [FeII] with SINFONI, suggesting a strongly shocked medium (see also Sect. 3.6).

\subsection{Carbon monoxide luminosity and $\mathrm{H}_{2}$ mass}

The mean intensity map is plotted in Fig. 4 (left). Since the galaxy is more extended than the primary beam, it is difficult to quantify the missing flux. We compare it to the central spectrum obtained with the $15 \mathrm{~m}$ single dish of the Swedish-ESO Submillimeter Telescope (SEST) in $\mathrm{CO}(1-0)$ and $\mathrm{CO}(2-1)$ over a $43^{\prime \prime}$ and $22^{\prime \prime}$ FoV, respectively. In Fig. 6, we display the total $\mathrm{CO}(3-2)$ spectrum integrated over the $18^{\prime \prime}$ FoV. Towards the central position, Bajaja et al. (1995) found a $\mathrm{CO}(2-1)$ spectrum peaking at $T_{A}^{*}=200 \mathrm{mK}$ with $F W H M=300 \mathrm{~km} \mathrm{~s}^{-1}$, yielding a total integrated flux of $1504 \mathrm{Jy} \mathrm{km} \mathrm{s}^{-1}$, in a beam of $22^{\prime \prime}$. Their beam is very similar to our FoV of $18^{\prime \prime}$. The flux comparison is relevant, since our FoV encompasses the entire nuclear ring, and the emission in this nuclear region corresponds to the strongest surface density at different wavelengths (Comerón et al. 2010; Ho et al. 2011; Li et al. 2011), as already discussed by Combes et al. (2014).

We assume a ratio of $r_{31}=T_{3-2} / T_{1-0}$ of 0.82 , typical for Seyfert galaxies (Mao et al. 2010) and a ratio $r_{21}=T_{2-1} / T_{1-0}$ compatible with 1 within the error bars. The latter is derived from the SEST $\mathrm{CO}(2-1) / \mathrm{CO}(1-0)$ observations in the galaxy center, by convolving the beam of $\mathrm{CO}(2-1)$ to $43^{\prime \prime}$, implying a 


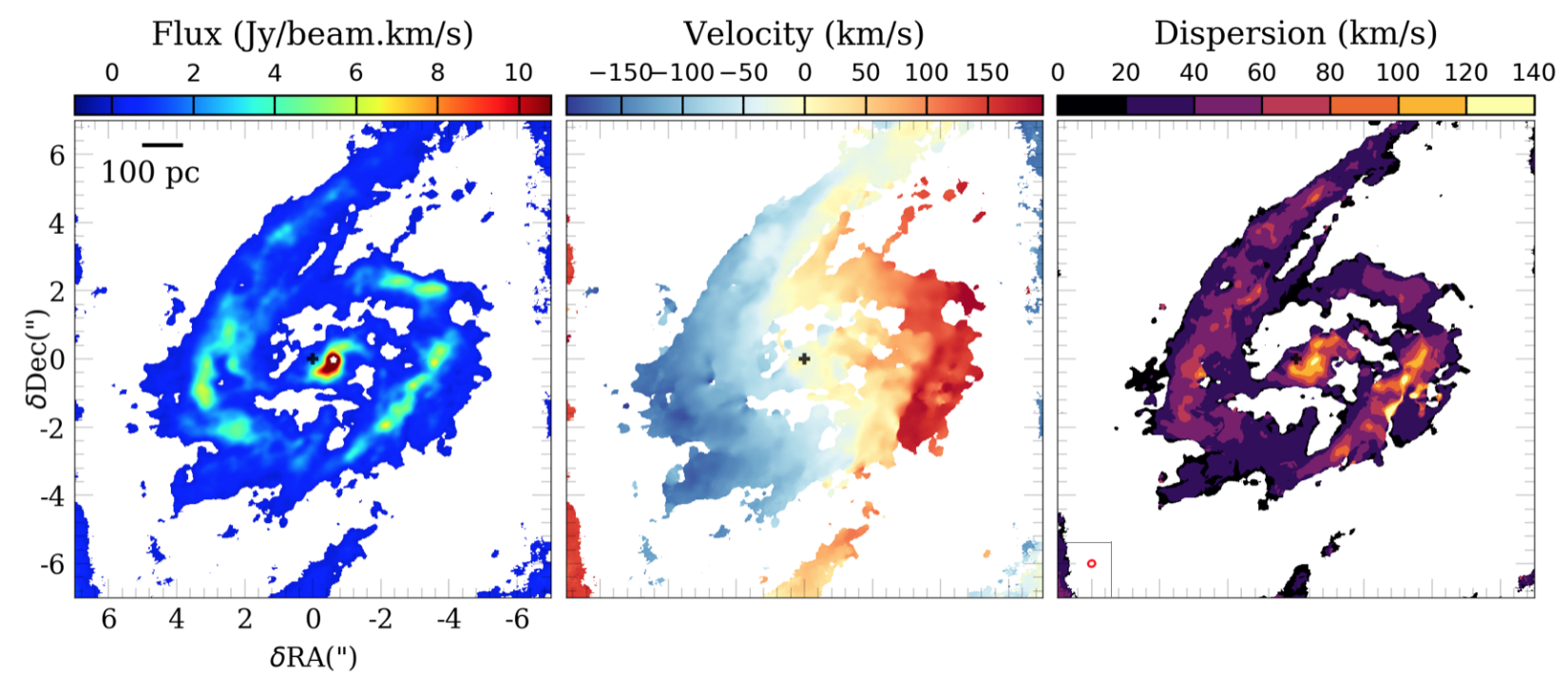

Fig. 4. $\mathrm{CO}(3-2)$ moment maps of NGC 613 in the central $14^{\prime \prime}(\sim 1.2 \mathrm{kpc})$ FoV. We show the integrated intensity map (0th moment, left), intensityweighted velocity map (first-moment, middle), and the intensity-weighted velocity dispersion map (second-moment, right). The black cross indicates the phase center and the white star the new adopted AGN position (in Table 1). The synthesised beam of $0^{\prime \prime} \cdot 21 \times 0^{\prime \prime} \cdot 19$ is shown in red in the bottom left corner of the second-moment map.

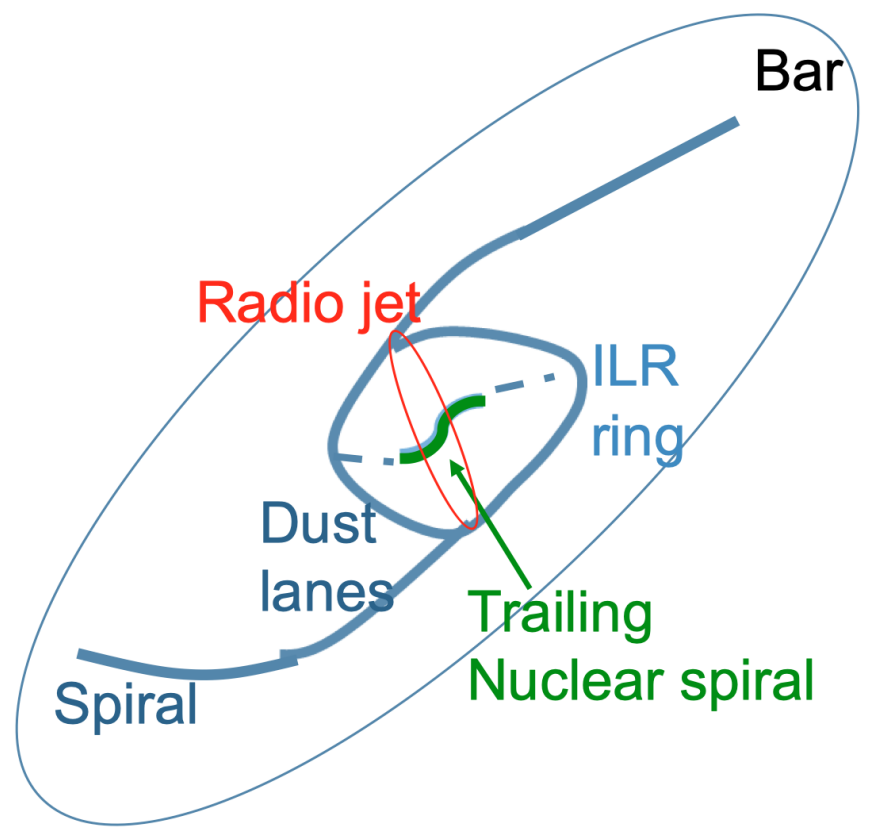

Fig. 5. Sketch of the main morphological features observed in the $\mathrm{CO}(3-2)$ emission in NGC 613. The orientation of the dust lanes follows the large scale bar. The filaments are shown as dashed lines between the ILR star-forming nuclear ring and the nuclear trailing spiral. The radio jet is also shown for comparison.

higher CO excitation at the center of NGC 613. This is expected for thermalized excitation and a dense molecular medium. In that case, the $\mathrm{CO}(3-2)$ flux should be higher than the $\mathrm{CO}(2-1)$, as we could presume the flux $S_{v} \propto v^{2}$ in the Rayleigh-Jeans approximation for gas at greater temperatures than $25 \mathrm{~K}$ and densities greater than $10^{4} \mathrm{~cm}^{-3}$. Using these values, the expected $\mathrm{CO}(3-2)$ intensity is $\sim 2266 \mathrm{Jy} \mathrm{km} \mathrm{s}^{-1}$ in a $22^{\prime \prime}$ beam. When integrated over the spectral range $\left(F W H M \sim 250 \mathrm{~km} \mathrm{~s}^{-1}\right)$, the integrated emission in our ALMA FoV of 18", shown in Fig. 6 is $1307 \mathrm{Jy} \mathrm{km} \mathrm{s}^{-1}$. Therefore, we should expect some missing flux by a factor up to $\sim 40-50 \%$, taking into account the uncertainties of the $r_{31}$ and $r_{21}$ ratios. We thus find a molecular

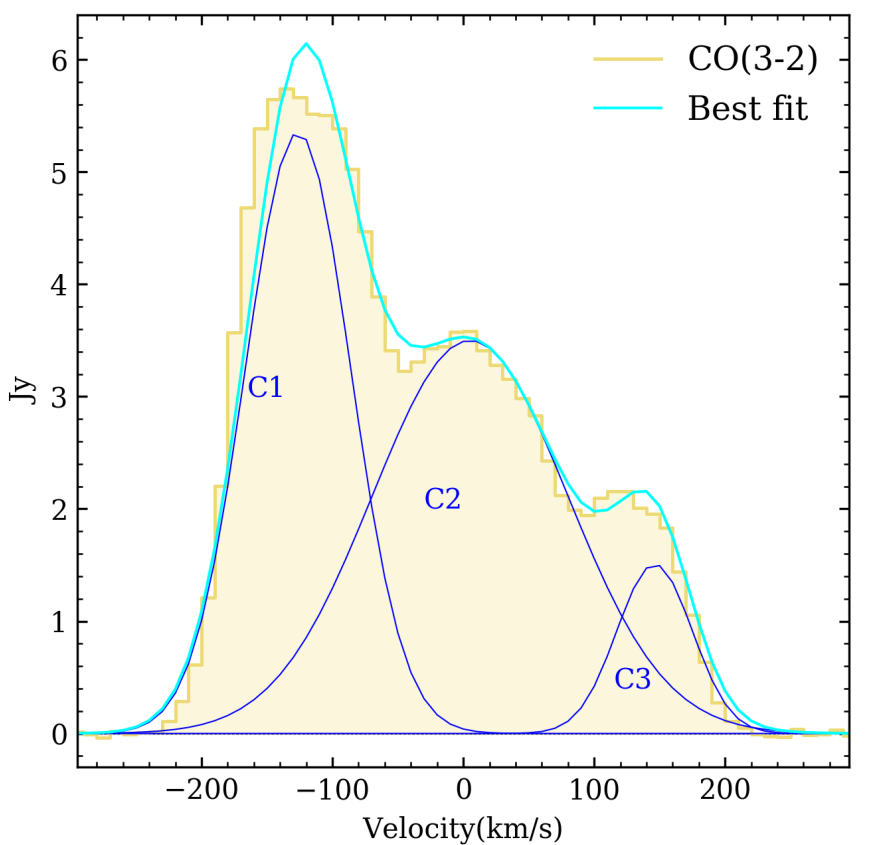

Fig. 6. Total $\mathrm{CO}(3-2)$ emission line profile integrated over the observed map, with a FoV of $18^{\prime \prime}$, after correction for primary beam attenuation. The light blue line is the result of the Gaussian fit with three velocity components (in dark blue); see Table 2.

mass of $5.6 \times 10^{8} M_{\odot}$ in our FoV, assuming thermally excited gas and a Milky-Way-like CO-to- $\mathrm{H}_{2}$ conversion factor of $2 \times$ $10^{20} \mathrm{~cm}^{-2} /\left(\mathrm{K} \mathrm{km} \mathrm{s}^{-1}\right)$; see for example Bolatto et al. (2013).

In comparison, the SEST CO(1-0) observations of Bajaja et al. (1995) give a total molecular mass of $3.8 \times 10^{9} M_{\odot}$ integrated over 33 pointing positions covering $\sim 120^{\prime \prime}$ along the galaxy, and $M_{\mathrm{H} 2} \approx 1.5 \times 10^{9} M_{\odot}$. in the central $43^{\prime \prime}$ beam. In the $22^{\prime \prime}$ beam, the SEST $\operatorname{CO}(2-1)$ spectrum, together with the $\mathrm{CO}(2-1) / \mathrm{CO}(1-0)$ ratio of 1 , gives a mass of $1.2 \times 10^{9} M_{\odot}$.

The total $\mathrm{CO}(3-2)$ emission line profile integrated over the observed map (FoV of 18") is shown in Fig. 6. We decomposed the spectrum in three components, $\mathrm{C} 1, \mathrm{C} 2$, and $\mathrm{C} 3$, and 
Table 2. Line fluxes.

\begin{tabular}{ccccc}
\hline \hline Line & $\begin{array}{c}S_{\mathrm{CO}(3-2)} \\
\left(\mathrm{Jy} \mathrm{km} \mathrm{s}^{-1}\right)\end{array}$ & $\begin{array}{c}V \\
\left(\mathrm{~km} \mathrm{~s}^{-1}\right)\end{array}$ & $\begin{array}{c}F W H M \\
\left(\mathrm{~km} \mathrm{~s}^{-1}\right)\end{array}$ & $\begin{array}{c}\mathrm{S}_{\text {peak }}(a) \\
(\mathrm{Jy})\end{array}$ \\
\hline $\mathrm{C} 1$ & $541.1 \pm 54.7$ & $-126.3 \pm 1.7$ & $95.0 \pm 3.6$ & 5.4 \\
$\mathrm{C} 2$ & $654.0 \pm 90.7$ & $5.2 \pm 5.0$ & $175.5 \pm 20.8$ & 3.5 \\
$\mathrm{C} 3$ & $109.1 \pm 27.7$ & $146.2 \pm 2.8$ & $68.0 \pm 8.4$ & 1.5 \\
\hline
\end{tabular}

Notes. Results of the Gaussian fits for the three velocity components (C1, C2 and C3) shown in Fig. 6. ${ }^{(a)}$ Peak flux.

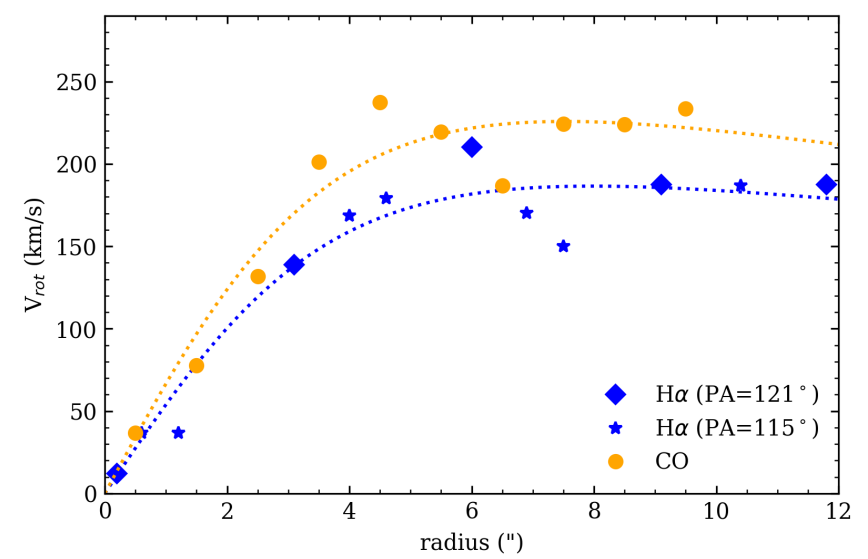

Fig. 7. Rotation curve of NGC 613. The orange circles represent the CO kinematics from our ALMA observations and the blue diamonds and stars are the $\mathrm{H} \alpha$ measurements by Burbidge et al. (1964), for a PA of $121^{\circ}$ and $115^{\circ}$, respectively. The dotted lines are the best fit assuming the gas to be on circular orbits in a plane, $v_{\mathrm{c}}=A r /\left(r^{2}+c^{2}\right)^{p / 2}$.

the results of the Gaussian fits for each component are displayed in Table 2. The total flux is $S_{\mathrm{CO}(3-2)}=1307 \pm 121 \mathrm{Jy} \mathrm{km} \mathrm{s}^{-1}$.

\section{4. $\mathrm{CO}(3-2)$ kinematics}

In Fig. 7 we show the rotation velocities deduced from the $\mathrm{CO}$ kinematics and the $\mathrm{H} \alpha$ rotation curve taken from Burbidge et al. (1964). As already pointed out, the dominant feature in the velocity field of the molecular gas appears to be due to circular rotation in the disk (middle panel of Fig. 4), and is consistent with the $\mathrm{H} \alpha$ kinematics. We find good agreement with the PA and inclination from optical studies, in the range of $\mathrm{PA}=111 \sim 124^{\circ}$ and $i=36 \sim 47^{\circ}$ (Burbidge et al. 1964; Blackman 1981; de Vaucouleurs et al. 1991), and therefore we adopted the values of $\mathrm{PA}=120^{\circ}$ and $i=41^{\circ}$ listed in Table 1 . We assume a simple model proposed by Bertola et al. (1991) for the rotation curve, assuming the gas is on circular orbits in the plane $v_{\mathrm{c}}=A r /\left(r^{2}+c^{2}\right)^{p / 2}$, where $A, c$, and $p$ are parameters of the model, and for $p=1$ the velocity curve is asymptotically flat and for $p=3 / 2$ the system has a finite total mass, and therefore we expect $1 \leq p \leq 3 / 2$. Figure 7 shows the result of fitting the radially averaged velocities to this model; the dotted lines represent the best fit for circular velocity, $v_{\mathrm{c}}$.

We can refine the above fit of the radially averaged velocities by fitting the entire velocity field with the same model by Bertola et al. (1991). The observed radial velocity at a position $(R, \Psi)$ on the plane of the sky can be described as

$$
v(R, \Psi)=v_{\text {sys }}+\frac{A R \cos \left(\Psi-\Psi_{0}\right) \sin (\theta) \cos ^{p}(\theta)}{\left\{R^{2}\left[\sin ^{2}\left(\Psi-\Psi_{0}\right)+\cos ^{2}(\theta) \cos ^{2}\left(\Psi-\Psi_{0}\right)\right]+c^{2} \cos ^{2}(\theta)\right\}^{p / 2}},
$$

where $\theta$ is the inclination of the disk (with $\theta=0$ for a face-on disk), $\Psi_{0}$ is the PA of the line of nodes, $v_{\text {sys }}$ is the systemic velocity, and $R$ is the radius. We used the tilted-ring model (Rogstad et al. 1974), which consists in dividing the velocity field into concentric rings in radii $\Delta r$, with each ring being allowed to have an arbitrary $v_{\mathrm{c}}, i$, and PA. For each radius, we can independently fit the parameters of Eq. (1) to the observed velocity field.

We show the results of the fitting of the tilted-ring to the velocity map in Fig. 8. We adopted a $\Delta r=0,3$, which corresponds to the deprojected resolution of our observations in the galaxy plane. In the right panel, we display the residuals after subtracting the Bertola et al. (1991) model from the velocity field. As can be seen, the model represents the observed velocity field relatively well, with no significant amplitudes in the residuals along the galaxy disk, except in the west part of the ring where there is an important contribution from noncircular motions. This region coincides with the contact point between the ring and the SE winding arm, and it is also probably perturbed by shocks, as suggested by the high-velocity dispersions in the molecular gas and an enhancement in the [FeII] emission (Fig. 13). These noncircular motions along the minor axis and on the winding spiral indicate streaming motions associated with inflow, if we assume both trailing perturbations and that the north side is the far side of the galaxy, as the NW arm is blueshifted and the SE arm is redshifted.

An additional method was used to derive the CO kinematics using the "3D-Based Analysis of Rotating Objects from Line Observations" ( ${ }^{3 D}$ BAROLO) software by Di Teodoro \& Fraternali (2015). ${ }^{3 D}$ BAROLO performs a 3D tilted-ring modeling of the emission line data cubes to derive the parameters that best describe the kinematics of the data. We ran ${ }^{3 \mathrm{D}} \mathrm{BAROLO}$ on the $\mathrm{CO}(3-2)$ data-cube in order to investigate non circular motions, since the code allows us to infer radial velocities in the fit of the rotation curves. We performed several tests running the code, varying the fixed and free parameters of the disk model, and the results that better reproduce the observed velocity field are found when fixing the PA, the inclination, and the central position to $120^{\circ}, 41^{\circ}$, and the position of the AGN, respectively. The best fit reveals radial components of the order of $v_{\text {rad }} \sim 20 \mathrm{~km} \mathrm{~s}^{-1}$ in the nuclear region and $v_{\text {rad }} \sim 20-100 \mathrm{~km} \mathrm{~s}^{-1}$ from the end of the circumnuclear ring at $4^{\prime \prime}$ up to $r \sim 7^{\prime \prime}$, corresponding to the streaming motions of the winding arms. Overall, we find that in the case of high-resolution ALMA observations, the results using ${ }^{3 \mathrm{D}} \mathrm{BAROLO}$ are relatively coherent with the $2 \mathrm{D}$ approach described above.

The position-velocity diagrams (PVDs) along the major $\left(\mathrm{PA}=120^{\circ}\right)$ and minor axis $\left(210^{\circ}\right)$ of NGC 613 are shown in Fig. 9. The best fit obtained with ${ }^{3 \mathrm{D}}$ BAROLO including radial velocity components is shown in dashed lines. We notice highly skewed kinematics in the center, with velocities $\gtrsim 200 \mathrm{~km} \mathrm{~s}^{-1}$ that cannot be described only by co-planar circular motions in the galaxy disk. Since the velocities in the very center $\left(\lesssim 0.5^{\prime \prime}\right)$ strongly deviate from the rotation curve pattern, we believe this is the signature of an outflow emanating from the AGN. We discuss the detection and properties of the molecular outflow in Sect. 4.

\subsection{Dense gas: $\mathrm{HCO}^{+}, \mathrm{HCN}$, and CS emission}

In ALMA band 3, dense gas is detected in various lines, namely $\mathrm{HCN}(1-0), \mathrm{HCO}^{+}(1-0)$ and $\mathrm{CS}(2-1)$, while $\mathrm{SiO}(2-1)$ was marginally detected at the edges of the radio jets, probably indicating the existence of shock regions related to the jets, as reported by Miyamoto et al. (2017). Along with $\mathrm{CO}(3-2)$, our 

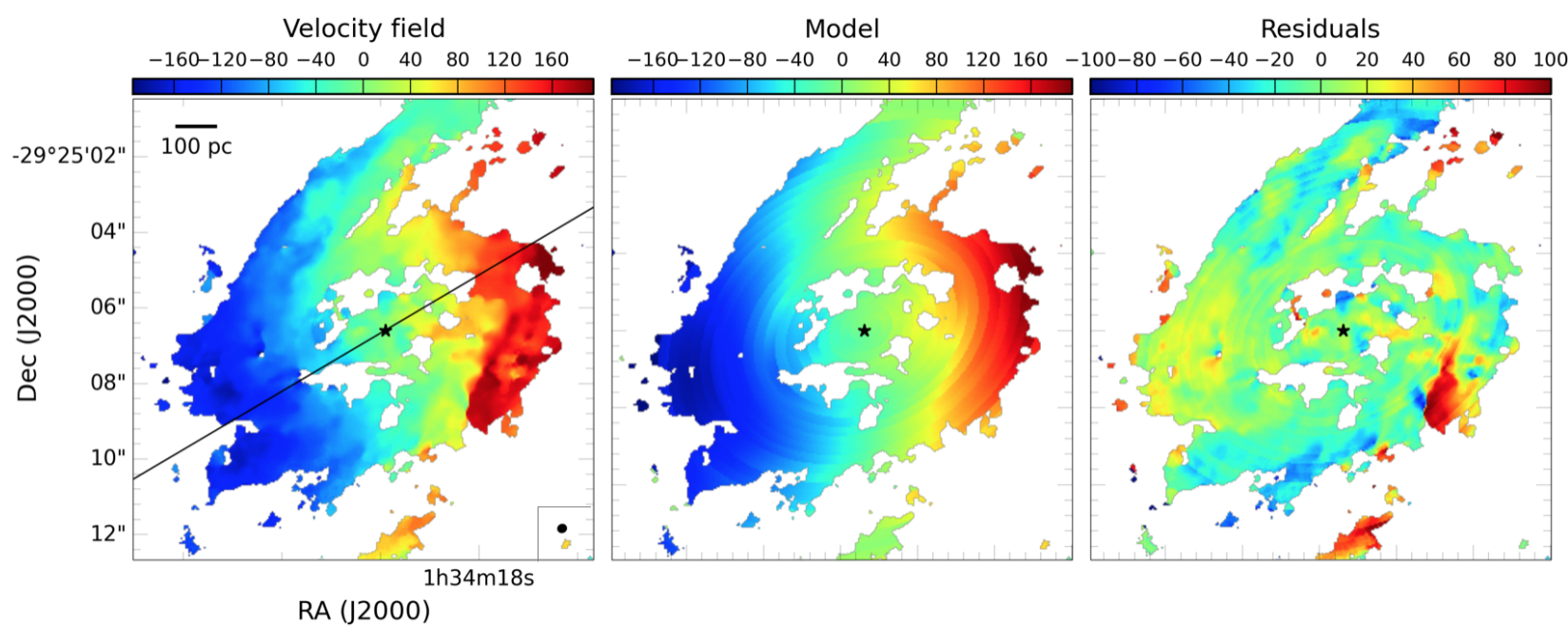

RA $(2000)$

Fig. 8. Left: velocity map of NGC 613 clipped at $>5 \sigma$. Middle: best-fit model using the tilted-ring approach for the Bertola et al. (1991) model. Right: residuals after subtracting the model from the data. The line in the left panel indicates the average estimated major axis $\mathrm{PA}=120^{\circ}$ and the black stars are the central positions adopted as the AGN position. The synthesized beam is shown as a black ellipse in the bottom right corner of the left panel.
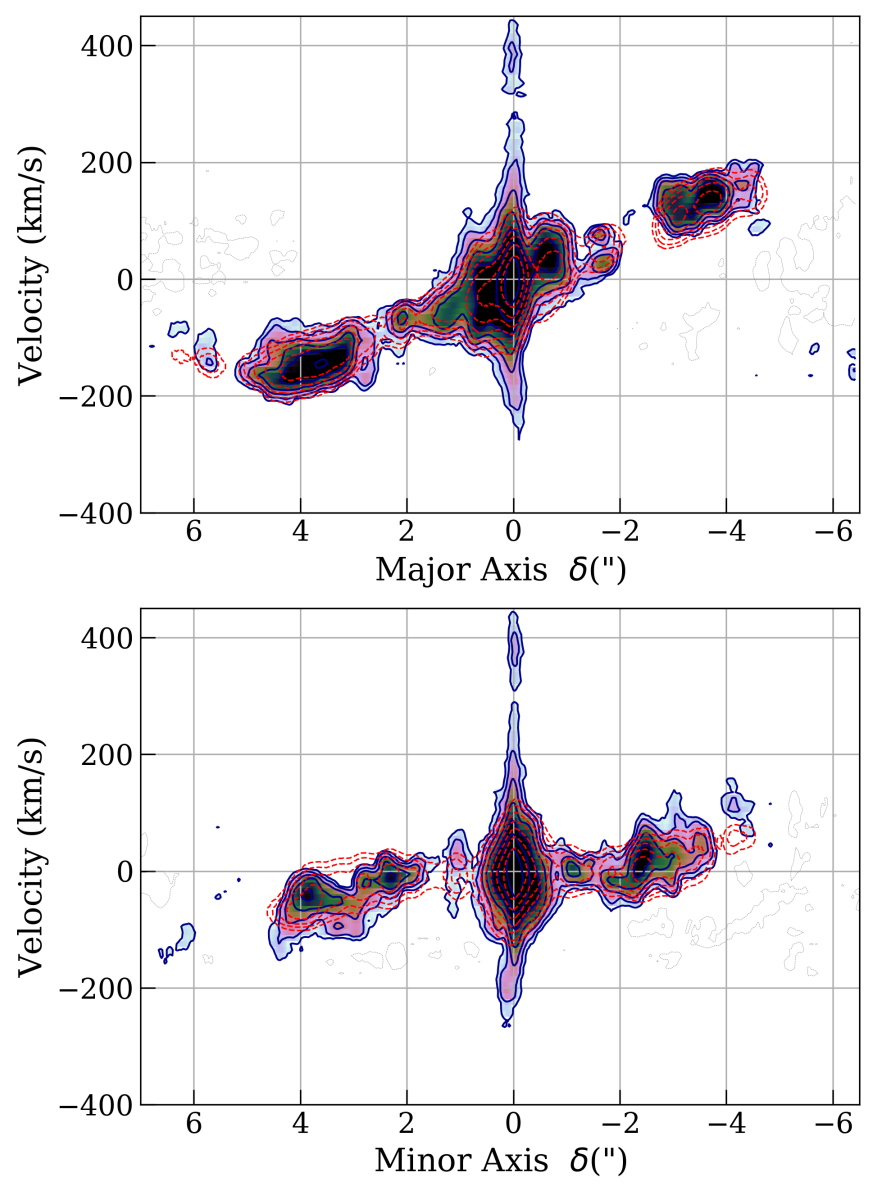

Fig. 9. Position-velocity diagrams of NGC 613 for the $\mathrm{CO}(3-2)$ emission along the major axis at a $\mathrm{PA}=120^{\circ}$ (top panel) and minor axis $\left(\mathrm{PA}=210^{\circ}\right.$, bottom $)$. We used a $00^{\prime \prime} 2$ slit width. The blue contours are from $1 \sigma$ to $64 \sigma$, and follow each other by factor-two multiplication. The red dashed lines are the best fit from ${ }^{3 \mathrm{D}} \mathrm{BAROLO}$. The emission around $v \sim 400 \mathrm{~km} \mathrm{~s}^{-1}$ corresponds to the isotope $\mathrm{H}^{13} \mathrm{CN}(4-3)$.

band 7 observations detected the $\mathrm{HCO}^{+}(4-3), \mathrm{HCN}(4-3)$, and CS(7-6) emission lines. The corresponding maps are displayed in Fig. 10, and the integrated spectra in Fig. 11. The CS(7-6) line is mainly detected in the center of the galaxy, while for $\mathrm{HCO}^{+}(4-3)$ and $\mathrm{HCN}(4-3)$, we detect stronger emission in the center but also some clumps along the star-forming ring. The dense gas tracers detected in the very center are interpreted as a molecular torus, as discussed in Paper I. The HCN line is about twice brighter than the $\mathrm{HCO}^{+}$line in the nuclear region, which is the typical value expected for an AGN (e.g., Kohno et al. 2005; Krips et al. 2008; Imanishi et al. 2016). The detection of these lines reveals the presence of dense gas, since the critical densities of the $\mathrm{HCO}^{+}(4-3), \mathrm{HCN}(4-3)$, and $\mathrm{CS}(7-6)$ transitions are $2.6 \times 10^{6}, 1.4 \times 10^{7}$, and $3.4 \times 10^{6} \mathrm{~cm}^{-3}$, respectively.

As already mentioned, NGC 613 exhibits different ionization mechanisms. Recently, spatially resolved Baldwin, Phillips, \& Terlevich (BPT) diagrams (Baldwin et al. 1981) were able to isolate the contributions from star formation, shock excitation, and AGN activity using optical line ratios, as studied with the Siding Spring Observatory Wide-Field Spectrograph (WiFeS) by Davies et al. (2017) and the European Southern Observatory Multi-unit Spectroscopic Explorer (MUSE) in Gadotti et al. (2019). Likewise, in the submillimeter domain, multi-line observations of higher $\mathrm{J}$ transitions are fundamental for the derivation of the chemical conditions of the molecular gas and the heating mechanisms (Viti et al. 2014; Imanishi et al. 2018). One useful extinction-free energy diagnostic tool in the centers of galaxies is the submillimeter-HCN diagram (Fig. 12) proposed by Izumi et al. (2016). The diagram uses the $\mathrm{HCN}(4-3) / \mathrm{HCO}^{+}(4-3)$ and $\mathrm{HCN}(4-3) / \mathrm{CS}(7-6)$ ratios to distinguish the dominant energy source exciting the molecular gas in galaxies, whether by an AGN (XDR or X-ray-dominated region) or star formation (PDR or photodissociation region). The authors suggest enhanced integrated intensity ratios in circumnuclear molecular gas around AGN compared to those in starburst galaxies (submillimeter HCN enhancement).

Thanks to our high-resolution ALMA observations, we are able to disentangle the emission coming from the nuclear region within the spiral trailing feature observed in the central $\sim 100 \mathrm{pc}$ and the contribution from a star-forming clump observed in all three molecular tracers at $\sim 250 \mathrm{pc}$ from the nucleus. The clump is indicated as a circle in Fig. 10. We measured the line intensity ratios $R_{\mathrm{HCN} / \mathrm{HCO}}^{+}$and $R_{\mathrm{HCN} / \mathrm{CS}}$ in these two regions (CND/AGN, for a central aperture of 0.3 and clump; listed in Table 3). 


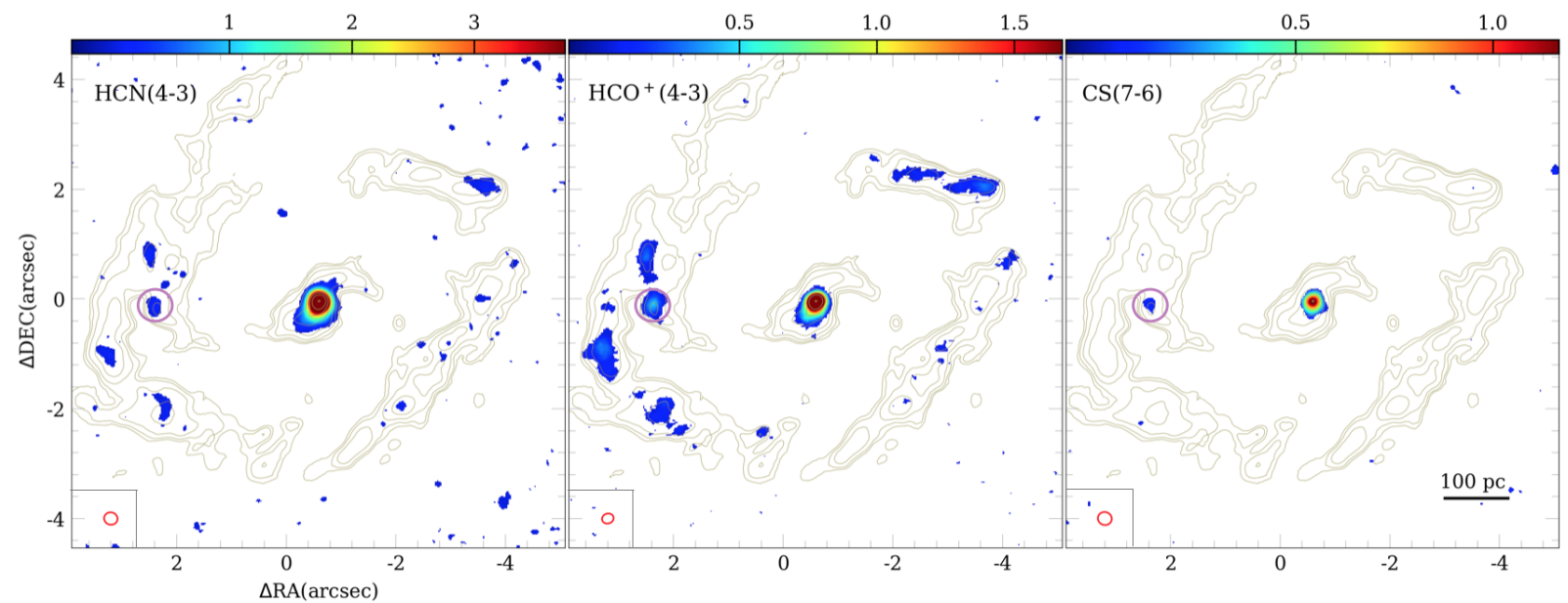

Fig. 10. Integrated intensity maps of the dense gas tracers $\mathrm{HCN}(4-3), \mathrm{HCO}^{+}(4-3)$, and $\mathrm{CS}(7-6)$ in the panels from left to right, with the $\mathrm{CO}$ contours overplotted. $\mathrm{HCN}$ and $\mathrm{HCO}^{+}$present clumpy emissions along a radius of $\sim 3^{\prime \prime}$, coinciding with the star-forming ring. All the dense gas tracers are detected in the very center as nuclear compact decoupled disks, or molecular tori as discussed in Paper I. The purple circles indicate the position of a clump detected in the $\mathrm{HCN}, \mathrm{HCO}^{+}$, and $\mathrm{CS}$ emission and used to calculate the ratios in the submillimeter diagram in Fig. 12. The synthesized beam sizes are shown in red in the bottom left corner of each panel.
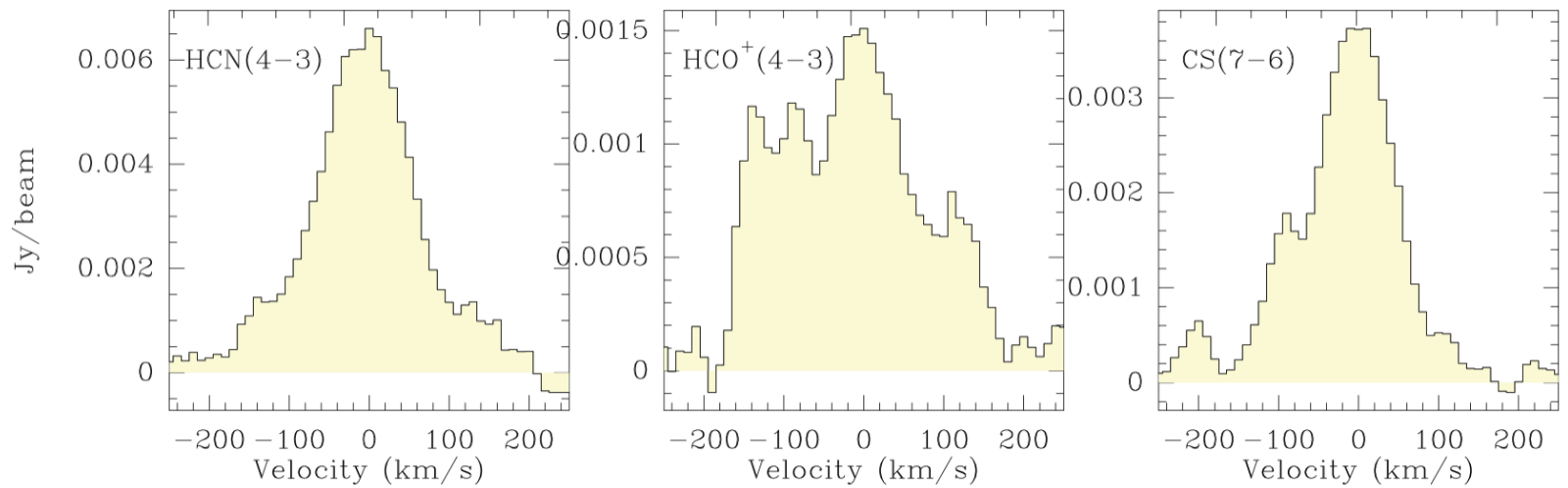

Fig. 11. $\mathrm{HCN}(4-3), \mathrm{HCO}^{+}(4-3)$, and $\mathrm{CS}(7-6)$ emission line profiles (from left to right) integrated along our FoV, using the mask from the zeromoment map in Fig. 10. The integrated values fitting a Gaussian are listed in Table 2.

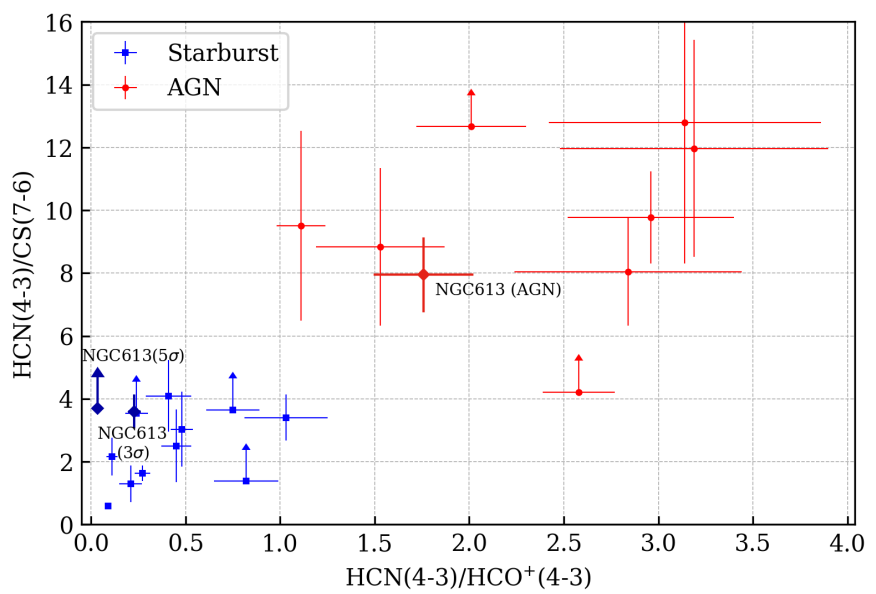

Fig. 12. Submillimeter-HCN diagram proposed by Izumi et al. (2016) for the high-resolution observations (spatial resolution $<500 \mathrm{pc}$ ) using

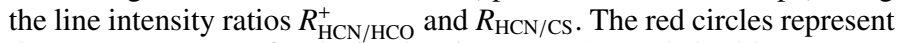
the measurements of galaxies hosting an AGN and the blue squares indicate the ratios found in starburst galaxies. We include the line ratios of NGC 613 (diamonds) measured in the CND, referred to here as AGN and in a clump detected $\sim 250 \mathrm{pc}$ northeast of the central position in all the dense tracers shown in Fig. 10.
Table 3. Line ratios: $R_{\mathrm{HCN} / \mathrm{HCO}}^{+}$and $R_{\mathrm{HCN} / \mathrm{CS}}$.

\begin{tabular}{ccc}
\hline \hline Region & $R_{\mathrm{HCN} / \mathrm{HCO}}^{+}$ & $R_{\mathrm{HCN} / \mathrm{CS}}$ \\
\hline AGN & $1.76 \pm 0.26$ & $7.95 \pm 1.19$ \\
Clump $(3 \sigma)$ & $0.23 \pm 0.03$ & $3.6 \pm 0.5$ \\
Clump $(5 \sigma)$ & $0.034 \pm 0.005$ & $>3.7 \pm 0.6$ \\
\hline
\end{tabular}

Notes. Ratios of the $\mathrm{HCN}, \mathrm{HCO}^{+}$, and $\mathrm{CS}$ lines in the $\mathrm{CND}$ region and in the clump shown in Fig. 10.

For a $5 \sigma$ threshold, the clump is barely detected in $\operatorname{CS}(7-6)$ emission, and we present the line ratios for a $3 \sigma$ clump detection and an upper limit for the $R_{\mathrm{HCN} / \mathrm{CS}}$.

The line ratios are plotted on the submillimeter diagram of Izumi et al. (2016), as displayed in Fig. 12. We find that the CND region lies in the AGN-dominated part of the diagram, while the clump in the star-forming ring of NGC 613 is indeed dominated by star formation. Ultimately, we do find that the nuclear region of NGC 613 presents line ratios that indicate excitation conditions typical of XDRs in the vicinity of AGN. However, the line ratios in the center could also be explained by shocks driven by the radio jet entraining the gas, as HCN can become enhanced in 

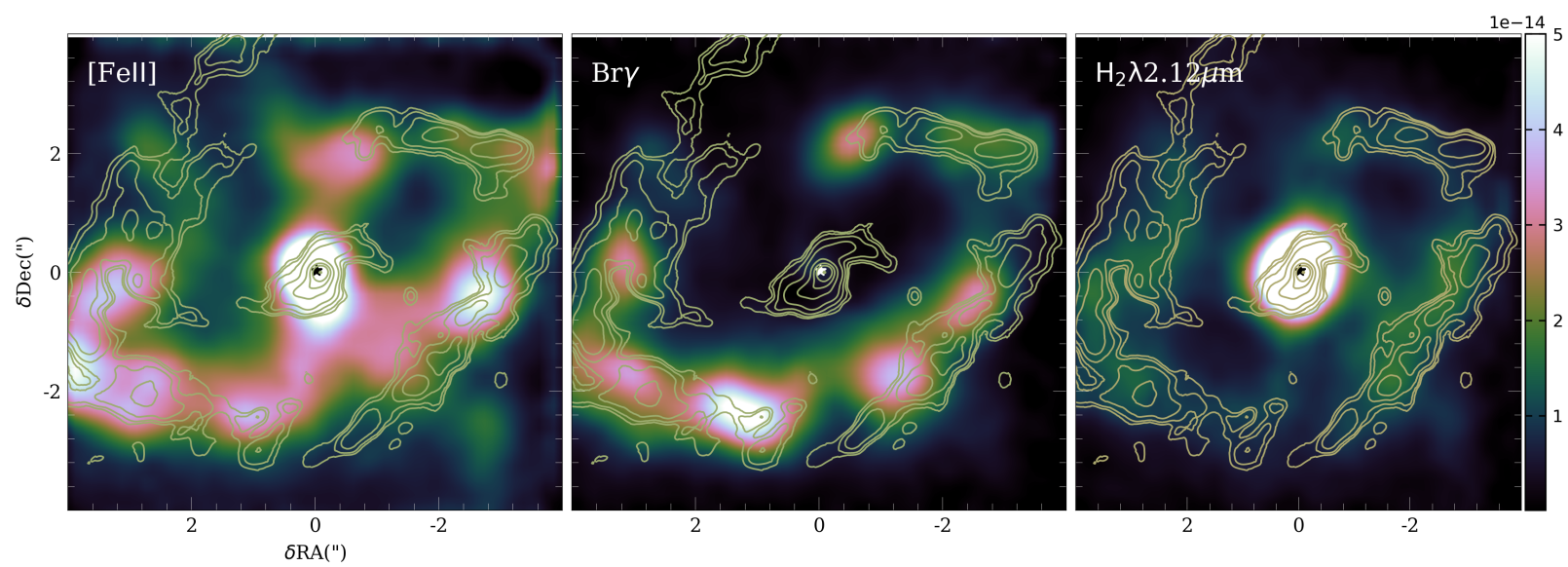

Fig. 13. Comparison between the $\mathrm{CO}(3-2)$ emission, shown in contours, with the [FeII] (left), $\mathrm{Br} \gamma$ (middle), and $\mathrm{H}_{2} \lambda 2.12 \mu \mathrm{m}$ (right) emission in the $8^{\prime \prime} \times 8^{\prime \prime}$ FoV of the SINFONI observations (Falcón-Barroso et al. 2014). The black and white star represents the AGN position listed in Table 1 .

shocks and this scenario is also suggested by the [FeII] enhancement discussed in Sect. 3.6.

\subsection{Comparison to the warm molecular and ionized gas}

In order to compare the $\mathrm{CO}(3-2)$ morphology with the ionized material and the warm molecular gas, we superposed the $\mathrm{CO}$ contours onto the NIR maps of [FeII], $\mathrm{Br} \gamma$, and $\mathrm{H}_{2} \lambda 2.12 \mu \mathrm{m}$ presented in Falcón-Barroso et al. (2014), shown in Fig. 13. There is a remarkable resemblance between the ionized and warm molecular gas and the $\mathrm{CO}$ emission along the star forming ring. The positions and ages of the hot spots in the ring suggest a "pearls on a string" scenario of evolution of star formation as proposed by Böker et al. (2008). In this scenario, star formation only occurs in particular overdense regions and the young clusters move along the ring, following the gas movement, and simultaneously age, resulting in an age gradient along the ring. The expected sequence of star formation was indeed observed in the southern part of the ring: the hottest stars are found near the contact point of the dust lanes, and then fewer hot stars are found along the ring (see Fig. 8 of Böker et al. 2008).

The $\mathrm{CO}(3-2)$ emission presents lower intensities in the starforming hot spots at the contact points of the winding arms and the ring, corresponding to the hottest and youngest star-clumps traced by the HeI emission in Fig. 8 of Böker et al. (2008). This anti-correlation can be expected between the SFR and the gas surface density on small scales $(<200 \mathrm{pc})$, since the gas has probably been consumed in the formation of new stars and the KennicuttSchimidt law (Schmidt 1959; Kennicutt 1998a) breaks down on scales of giant molecular clouds (Onodera et al. 2010). However, we do observe some dense clumps in $\mathrm{HCO}^{+}$and $\mathrm{HCN}$ emission, corresponding to other less hot regions of HeI emission (blue regions in their Fig. 8), indicating that some dense material is still being consumed in the southeast and northern part of the ring.

At the center, the nuclear spiral corresponds to the massive reservoir of the bright warm $\mathrm{H}_{2}$ and [FeII], while contrasting with the weak emission in $\mathrm{Br} \gamma$. Indeed, as discussed by FalcónBarroso et al. (2014), the high [FeII]/Br $\gamma$ ratio in the center indicates that excitation is dominated by shocks and photoionization in the nucleus, and follows the correlation between the strength of the [FeII] and $6 \mathrm{~cm}$ radio emission in Seyfert galaxies (Forbes \& Ward 1993).

The high values of the ratio $[\mathrm{FeII}] / \mathrm{Br} \gamma=17.7$ in the nucleus of NGC 613 are not typical of starburst galaxies, where we expect the ratio to be in the range $0.5-2$ (Colina et al. 2015) and the [FeII]

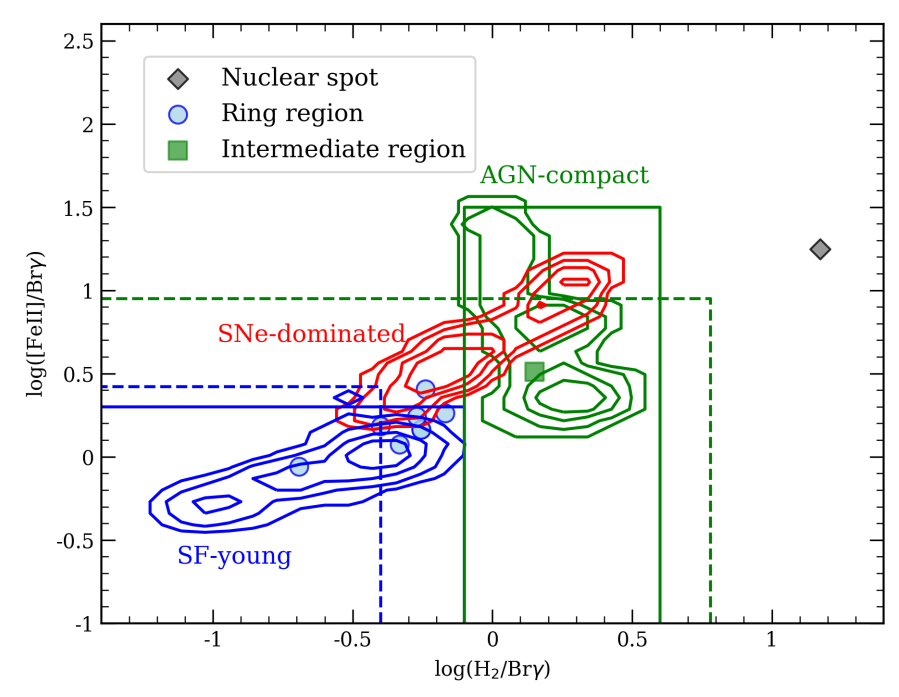

Fig. 14. Diagnostic diagram with the NIR emission line ratios $\mathrm{H}_{2}(1-0)$ $\mathrm{S}(1) \lambda 2.122 \mu \mathrm{m} / \mathrm{Br} \gamma$ and $[\mathrm{Fe} \mathrm{II}] \lambda 1.644 \mu \mathrm{m} / \mathrm{Br} \gamma$ proposed by Colina et al. (2015). Apertures in the circumnuclear ring are shown with blue circles and the aperture from the nuclear region is shown by a gray diamond. Contours denote regions for young star formation, supernovae, and compact AGN, while solid lines denote upper limits for young star formation and AGN, both derived from integral field spectroscopy data (Colina et al. 2015). Dashed lines denote upper limits for star formation and AGN derived from slit spectroscopy (Riffel et al. 2013).

emission originates in supernova-driven shocks. Indeed, the large ratio in the nucleus is similar to those found in AGN, indicating that the most likely mechanism for the production of [FeII] emission is shock excitation from the radio jets and/or supernova remnants typical of Seyfert galaxies (Rodríguez-Ardila et al. 2004). It is well-known that X-ray emission, which is dominant in Seyferts, can penetrate deeply into atomic gas and create extended partly ionized regions where [FeII] can be formed. Models presented by Alonso-Herrero et al. (1997) show that X-rays are able to explain $[\mathrm{FeII}] / \mathrm{Br} \gamma$ ratios of up to $\sim 20$, in agreement with the values observed in the NGC 613 nucleus.

Colina et al. (2015) developed a 2D diagnostic diagram using integral field spectrograph data to characterize line-emitting regions. The diagnostic uses the line ratios $\mathrm{H}_{2}(1-0) \mathrm{S}(1)$ $\lambda 2.122 \mu \mathrm{m} / \mathrm{Br} \gamma$ and [Fe II] $\lambda 1.644 \mu \mathrm{m} / \mathrm{Br} \gamma$. Using this method, these latter authors found that young star-forming regions, older supernova-dominated regions, and the compact AGN-dominated 
region occupy different areas in the line-ratio space. We show the NIR diagnostic diagram proposed by Colina et al. (2015) in Fig. 14. We use the line emission listed in Tables 1 and 2 of Falcón-Barroso et al. (2014) for different apertures: one in the nucleus, seven along the circumnuclear ring (spots 1 to 7), and one aperture between the ring and the nucleus (spot 8; see Fig. 1 of Falcón-Barroso et al. 2014). Placing the line ratios for the different apertures in the diagrams, we note that all spots in the circumnuclear ring are located in the regions of young star formation or SNe-dominated stellar populations. The spot in the intermediate region has higher line ratios, and is shifted into the compact AGN region. On the other hand, the nucleus of NGC 613 takes its place in the shock ionized LINER regime, where we expect $\mathrm{H}_{2} / \mathrm{Br} \gamma$ ratios greater than six (Mazzalay et al. 2013; Riffel et al. 2013). As suggested by Falcón-Barroso et al. (2014), the enhancement of the $\mathrm{H}_{2}$ emission in the nucleus is possibly due to the interaction with the radio jet. The high value of $\mathrm{H}_{2} / \mathrm{Br} \gamma$ measured in the nucleus of NGC 613 is consistent with a LLAGN Seyfert/LINER composite, which is strongly influenced from shock heating.

\section{Molecular outflow}

\subsection{The $C O(3-2)$ kinematics of the outflow}

The PVDs along the major and minor axes of the galaxy shown in Fig. 9 illustrate the highly skewed kinematics in the central part of NGC 613. The nuclear region contains gas reaching velocities up to $\sim \pm 300 \mathrm{~km} \mathrm{~s}^{-1}$ in projection, which is much higher than the rest of the nuclear disk gas, as we can see in the middle panel of Fig. 4.

Examination of the individual spectra at pixels around the AGN position reveals conspicuous blue- and redshifted wings in all the spectra within a radius of $r \sim 0.3^{\prime \prime}$. These broad line wings are characteristic of gas ejection out of equilibrium; in this case an indication of a molecular outflow. The integrated spectrum extracted in a central circular aperture of $0.28^{\prime \prime}(\sim 23 \mathrm{pc})$ is shown in Fig. 15. We can see a bump in the emission around $v \sim 400 \mathrm{~km} \mathrm{~s}^{-1}$, which corresponds to the isotope $\mathrm{H}^{13} \mathrm{CN}(4-3)$.

One might question whether the outflow signature could instead arise from a central mass able to induce such fast rotation in the center. If we assume that these high-velocity components are within the SoI, in a radius equivalent to our nuclear aperture of $\sim 0.3^{\prime \prime}=25 \mathrm{pc}$, or about $r=38 \mathrm{pc}$ in the galaxy plane, a massive black hole located in the center should have a mass of at least $M_{\mathrm{BH}}=\mathrm{v}^{2} \mathrm{R} / \mathrm{G}$ for the rotational velocity in the galaxy plane $v=460 \mathrm{~km} \mathrm{~s}^{-1}$ (corresponding to $\pm 300 \mathrm{~km} \mathrm{~s}^{-1}$ projected velocities), or $M_{\mathrm{BH}}=1.9 \times 10^{9} M_{\odot}$. This value is about two orders of magnitude higher than the values reported in the literature; for example, $M_{\mathrm{BH}}=7.4 \times 10^{6} M_{\odot}$ derived by Davis et al. (2014) using the spiral pitch angle and $M_{\mathrm{BH}}=4 \times 10^{7} M_{\odot}$ using the central stellar velocity dispersion by van den Bosch (2016). In Paper I, we derived a BH mass of $M_{\mathrm{BH}}=3.7 \times 10^{7} M_{\odot}$ within the SoI of $50 \mathrm{pc}$, and for the whole NUGA sample the values derived tend to follow the pseudo-bulge region in the $M_{\mathrm{BH}}-\sigma$ plane (Ho \& Kim 2014).

Furthermore, if these high-velocity features were due to the rotation, they would not be observed along the minor axis of the galaxy; however, the PVDs in Fig. 9 clearly show a gradient of high-velocity emission along the minor axis that cannot be explained as being due to co-planar rotation. Could these features be a signature of inflowing gas? We cannot exclude a priori that the high-velocity components are due to a coplanar inflow, since the blueshifted gas is observed in the north and the

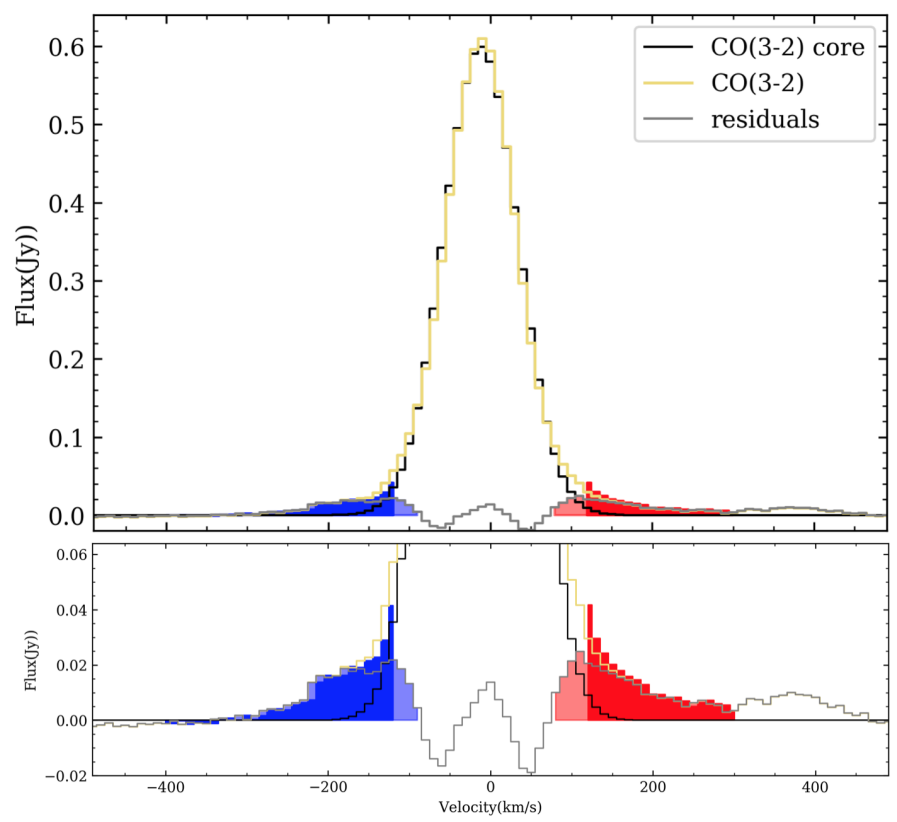

Fig. 15. Top panel: nuclear $\mathrm{CO}(3-2)$ spectrum in yellow, extracted in a 0 '28 region. We fit a Gaussian that takes into account the main disk (or "core") contribution to the $\mathrm{CO}$ emission and subtract this from the observed spectrum (residuals in gray). The regions considered in the computation of the molecular outflow properties in the blue and red wings are shown in color. Bottom panel: zoom view of the blue $(-400$ to $-120 \mathrm{~km} \mathrm{~s}^{-1}$ ) and red (120 to $300 \mathrm{~km} \mathrm{~s}^{-1}$ ) wings. The red wing is integrated only up to $+300 \mathrm{~km} \mathrm{~s}^{-1}$ to avoid the contamination from the $\mathrm{H}^{13} \mathrm{CN}$ emission at $\sim 400 \mathrm{~km} \mathrm{~s}^{-1}$.

redshifted gas is seen in the south. If the far side of the galaxy is the north side, this gradient could be explained as an inflow. However, the deprojected velocities onto the galaxy disk would be $v \sim 300 / \sin (i) \sim 460 \mathrm{~km} \mathrm{~s}^{-1}$; the order of magnitude of the co-planar inflow would thus be too large, and therefore we can exclude this hypothesis.

Additionally, in Fig. 16 we show the contours for the blue and red wing emission, and we see that the contours overlap, the blue component arises in the northern part of the nucleus, and the red component in the south. Due to the small size of the outflow, we can barely resolve each wing contribution. However, there is an indication that they do not follow the rotation pattern of the mean velocity field. The direction of velocities are also opposite to what is found in the molecular torus (cf. Paper I).

We compare the molecular to the radio emission observed with the Karl G. Jansky Very Large Array (VLA) at $4.86 \mathrm{GHz}$ (Hummel \& Jorsater 1992) in Fig. 16. The VLA radio jet emission is shown in the right panel with the molecular outflow blue and red wing emission contours overplotted. The molecular outflow emission coincides with the central blob of the radio jet and appears to be aligned with the orientation of the radio jet at $\mathrm{PA}=12^{\circ}$.

In order to derive conservative values for the flux related to the outflow in the broad emission, instead of fitting one Gaussian component for the "core" and one broad component for the outflow (including some low-velocity emission that might not be associated with the outflowing material), we have taken two different approaches. First, we fitted a Gaussian to the nuclear spectrum to take into account the contribution of the main rotating disk (black line in Fig. 15). The residual spectrum is shown by the gray line, after subtracting the core contribution from the $\mathrm{CO}$ spectrum. We then integrated the contribution of the blue and 

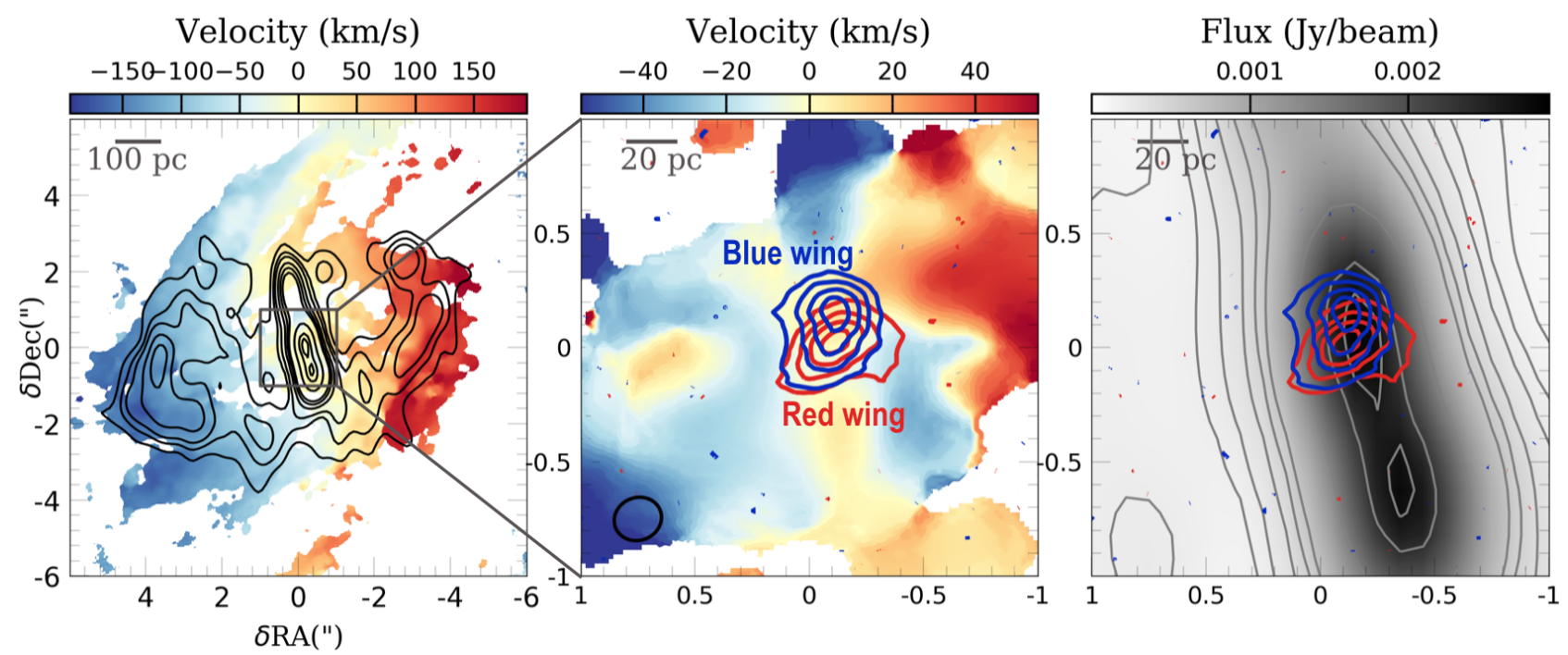

Fig. 16. Left: velocity distribution of the $\mathrm{CO}(3-2)$ emission, as presented in Fig. 4, with the VLA radio contours at 4.86 GHz over-plotted. Middle: $2^{\prime \prime} \times 2^{\prime \prime}$ zoom of the velocity distribution and the emission contours of the blue and red wings shown in Fig. 15. The synthesized beam is shown in black in the bottom left corner. Right: grayscale and contours of the $4.86 \mathrm{GHz}$ radio emission and the contours of the blue and red wings. The direction of molecular outflow is aligned with the radio jet, and spatially corresponds to the central blob of the radio map.

Table 4. Gaussian fit in the nuclear spectrum.

\begin{tabular}{|c|c|c|c|c|}
\hline Component & $\begin{array}{c}S_{\mathrm{CO}} \\
\left(\mathrm{Jy} \mathrm{km} \mathrm{s}^{-1}\right)\end{array}$ & $\begin{array}{l}\text { Position } \\
\left(\mathrm{km} \mathrm{s}^{-1}\right)\end{array}$ & $\begin{array}{l}F W H M \\
\left(\mathrm{~km} \mathrm{~s}^{-1}\right)\end{array}$ & $\begin{array}{l}S_{\text {peak }} \\
(\mathrm{mJy})\end{array}$ \\
\hline \multirow[t]{2}{*}{ Core $^{(a)}$} & $68.5 \pm 1.1$ & $-11.8 \pm 0.4$ & $107.2 \pm 0.9$ & 600 \\
\hline & $\begin{array}{c}S_{\mathrm{CO}} \\
\left(\mathrm{Jy} \mathrm{km} \mathrm{s}^{-1}\right)\end{array}$ & \multicolumn{2}{|c|}{$\begin{array}{l}\text { Velocity range } \\
\left(\mathrm{km} \mathrm{s}^{-1}\right)\end{array}$} & $\begin{array}{l}S_{\text {peak }} \\
(\mathrm{mJy})\end{array}$ \\
\hline \multicolumn{5}{|c|}{ INTEGRATED RESIDUAL WINGS } \\
\hline Blue & $2.63 \pm 0.8$ & \multirow{2}{*}{\multicolumn{2}{|c|}{$\begin{array}{l}{[-400,-90]} \\
{[+80+300]}\end{array}$}} & 21.8 \\
\hline Red & $2.62 \pm 0.9$ & & & 24.9 \\
\hline \multicolumn{5}{|c|}{ INTEGRATED RESIDUAL WINGS } \\
\hline Blue & $2.3 \pm 0.8$ & {$[-400,120]$} & $41.5^{(b)}$ & \\
\hline Red & $2.3 \pm 0.9$ & {$[+120,+300]$} & $41.7^{(b)}$ & \\
\hline
\end{tabular}

Notes. ${ }^{(a)}$ Results of the Gaussian fit for the main disk contribution of the nuclear spectrum extracted in a $r=0.28^{\prime \prime}$ aperture shown in Fig. 15.

${ }^{(b)}$ In this case we assumed that $S_{\text {peak }}$ is the maximum flux in the nuclear spectrum within the selected velocity range of the blue and red wings.

red regions in the residual spectrum. The results of the fit of the main core and blue and red wings in the residuals are listed in Table 4. The derived molecular mass corresponding to the main disk component inside the radius of $23 \mathrm{pc}$ is $4.8 \times 10^{7} M_{\odot}$, which agrees with the mass of the $14 \mathrm{pc}$ molecular torus of $3.9 \times 10^{7} \mathrm{M}_{\odot}$ found in Paper I (using the same conversion factor and $r_{31}$ as in Paper I).

The second approach consists in creating moment maps only taking the velocity channels from -400 to $-120 \mathrm{~km} \mathrm{~s}^{-1}$ for the blue component and from $+120 \mathrm{~km} \mathrm{~s}^{-1}$ to $300 \mathrm{~km} \mathrm{~s}^{-1}$ for the red wing. We try to avoid the contribution from the $\mathrm{H}^{13} \mathrm{CN}$ by limiting the velocity channels up to $300 \mathrm{~km} \mathrm{~s}^{-1}$. We display the blue and red wing contour maps in Fig. 16, superimposed over the intensity weighted velocity map of the original CO map, which represents the mean velocity pattern. The integrated fluxes corresponding to velocity intervals of the red and blue maps are also listed in Table 4. The peak temperatures of the blue and red wings represent $\sim 5 \%$ of the peak of the main core component, however they are still detected with a $50 \sigma$ significance in the case of the wings in the residuals.

The maximal velocities of the red and blue components are up to about $\pm 300 \mathrm{~km} \mathrm{~s}^{-1}$ in projection $\left(\sim 460 \mathrm{~km} \mathrm{~s}^{-1}\right.$ if in the galaxy plane, $i=41^{\circ}$ ). Given their location near the nucleus, we tentatively interpret these high-velocity features as the two sides of an outflow. Globally, these features represent as much as $\lesssim 8 \%$ of the total molecular emission in the nuclear ring region.

\subsection{CO-to- $\mathrm{H}_{2}$ conversion in the nuclear region of NGC613}

From the integrated flux, $S_{\mathrm{CO}} \Delta V\left(\mathrm{Jy} \mathrm{km} \mathrm{s}^{-1}\right)$, listed in Table 4, we can derive the molecular mass involved in the outflow using the equation from Solomon \& Vanden Bout (2005):

$L_{\mathrm{CO}}^{\prime}\left(\mathrm{K} \mathrm{km} \mathrm{s}^{-1} \mathrm{pc}^{-2}\right)=3.25 \times 10^{7} \frac{S_{\mathrm{CO}} \Delta V}{1+z}\left(\frac{D_{\mathrm{L}}}{v_{\text {rest }}}\right)^{2}$,

where $v_{\text {rest }}=345.796 \mathrm{GHz}$, and $D_{\mathrm{L}}$ is the luminosity distance in Mpc. The molecular mass, including helium, is then derived from $\mathrm{M}\left(\mathrm{H}_{2}\right)=\alpha_{\mathrm{CO}} L_{\mathrm{CO}}^{\prime} \mathrm{r}_{13}$ (Tacconi et al. 2013). This implies a molecular mass of $M_{\text {out }}=1.9-2.2 \times 10^{6} M_{\odot}$. This mass was obtained using $r_{31}=0.82$, a luminosity distance of 17.2 Mpc, and the standard Galactic CO-to- $\mathrm{H}_{2}$ conversion factor $\left(\alpha_{\mathrm{CO}, \mathrm{MW}}=4.36 M_{\odot}\left(\mathrm{K} \mathrm{km} \mathrm{s}^{-1} \mathrm{pc}^{2}\right)^{-1}\right.$ Dame et al. 2001 ; Bolatto et al. 2013). However, this mass could be an upper limit if the flow is made of more diffuse optically thin gas.

The standard $\alpha_{\mathrm{CO}}=4.36$ for the Milky Way is the recommended value to use in the inner disks of galaxies. However, several observational works (e.g., Israel 2009a,b; Sandstrom et al. 2013) found that in the center of galaxies $(R \lesssim 1 \mathrm{Kpc})$ the conversion $X_{\mathrm{CO}}$ can be up to three to ten times lower than $X_{\mathrm{CO}, \mathrm{MW}}$. As pointed out by Bolatto et al. (2013), the recommended value to be applied in galaxy centers is $\alpha_{\mathrm{CO}, \text { cen }} \sim \frac{1}{4} \alpha_{\mathrm{CO}, \mathrm{MW}}$, with a 0.3 dex uncertainty. In our case, the masses involved in the outflow would be four times lower, that is, in the range of $M_{\text {out }}=$ $4.8-5.5 \times 10^{5} M_{\odot}$, providing a more conservative estimative of the mass.

The assumption of a smaller $\alpha_{\mathrm{CO}}$ has already been discussed in the literature. In the case of the outflow in NGC 1068, the 
$\frac{1}{4} \alpha_{\mathrm{CO}, \mathrm{MW}}$ factor was also assumed (García-Burillo et al. 2014), in agreement with the Large Velocity Gradient (LVG) approximation analysis of the $\mathrm{CO}$ line ratios in the central region of this galaxy (Usero et al. 2004). Another example is the molecular outflow detected in M 51, where the authors assumed $\alpha_{\mathrm{CO}}=$ $\frac{1}{2} \alpha_{\mathrm{CO}, \mathrm{MW}}$ (Querejeta et al. 2016; Matsushita et al. 2007). In a study of molecular gas excitation in the jet-driven winds of IC 5063, Dasyra et al. (2016) found that the outflowing molecular gas is partly optically thin, implying a $\alpha_{\mathrm{CO}}$ that is one order of magnitude smaller than that of the Galaxy.

Most molecular outflows are detected in ultra-luminous infrared galaxies (ULIRGs; Cicone et al. 2014). Therefore, the CO-to- $\mathrm{H}_{2}$ conversion factor usually assumed in the literature is $\alpha_{\mathrm{CO}}=0.8$, approximately five times lower than the Milky Way factor $\alpha_{\mathrm{CO}, \mathrm{MW}}$. Since NGC 613 has a rather moderate IR luminosity $\left(L_{\mathrm{IR}}=3 \times 10^{10} L_{\odot}\right)$, there is no reason a priori to adopt the lower factor applied to ULIRGs. In fact, we would like to highlight that uncertainties in $\alpha_{\mathrm{CO}}$ impact the comparison of scaling factors between outflows and host galaxies properties (e.g., Fiore et al. 2017; Fluetsch et al. 2019) by a factor of approximately five.

\subsection{Mass outflow rate}

To estimate the mass outflow rate, along with the observational quantities (outflow mass $M_{\text {out }}$, size $R_{\text {out }}$ and velocity $v_{\text {out }}$ ), we need to assume a certain geometry. Following Fiore et al. (2017) and Cicone et al. (2014), for a spherical or multi-conical geometry, in which the outflowing clouds are uniformly distributed along the flow, the mass outflow rate $\dot{M}_{\text {out }}$ can be calculated as:

$\dot{M}_{\text {out }}=3 v_{\text {out }}\left(M_{\text {out }} / R_{\text {out }}\right)$.

If instead we assume a time-averaged thin expelled shell geometry (Rupke et al. 2005b), also adopted in the study of molecular outflows in the local Universe (Veilleux et al. 2017; Fluetsch et al. 2019), we have

$\dot{M}_{\text {out }}=v_{\text {out }}\left(M_{\text {out }} / R_{\text {out }}\right)$,

which corresponds to the outflow mass averaged over the flow timescale, $t_{\text {flow }}=\frac{R_{\text {out }}}{v_{\text {out }}}$. The difference in the mass loading factor between the two proposed scenarios for the outflow geometry is a factor three times larger in the multi-conical/spherical description.

In the following estimates, we use Eq. (4) to derive more conservative outflow energetics, since the observations cannot constrain the geometry, and we are not able to favor one scenario over the other.

As discussed in Sect. 4.1, the outflow is found in a region of $R_{\text {out }}=0,28(\sim 23 \mathrm{pc})$ and here we consider the maximum projected velocity of the wings, $v_{\text {out }}=300 \mathrm{~km} \mathrm{~s}^{-1}$. If the outflow direction is between the observer line of sight and the galaxy plane, even assuming the maxima projected velocities, the de-projected velocities will encompass the adopted value, and therefore $v_{\text {out }}=300 \mathrm{~km} \mathrm{~s}^{-1}$ is a conservative value. The flow timescale is consequently $t_{\text {flow }} \sim 10^{4} \mathrm{yr}$, which is comparable to the timescales of the $\mathrm{BH}$ growth-burst episodes of nuclear activity, with a duration of $10^{4-5} \mathrm{yr}$ (Wada 2004).

For an outflow mass of $M_{\text {out }}=(1.9-2.2) \times 10^{6} M_{\odot}$ (assuming the standard $\alpha_{\mathrm{CO}, \mathrm{MW}}$ ), we find a mass outflow rate of $\dot{M}_{\text {out }}=$ $(25-29) M_{\odot} \mathrm{yr}^{-1}$. If instead, we use the mass derived assuming the typical values for galaxy centers, $\alpha_{\mathrm{CO}}=\frac{1}{4} \alpha_{\mathrm{CO}, \mathrm{MW}}$, we find that the mass load rate is $\dot{M}_{\text {out }} \sim 7 M_{\odot} \mathrm{yr}^{-1}$.

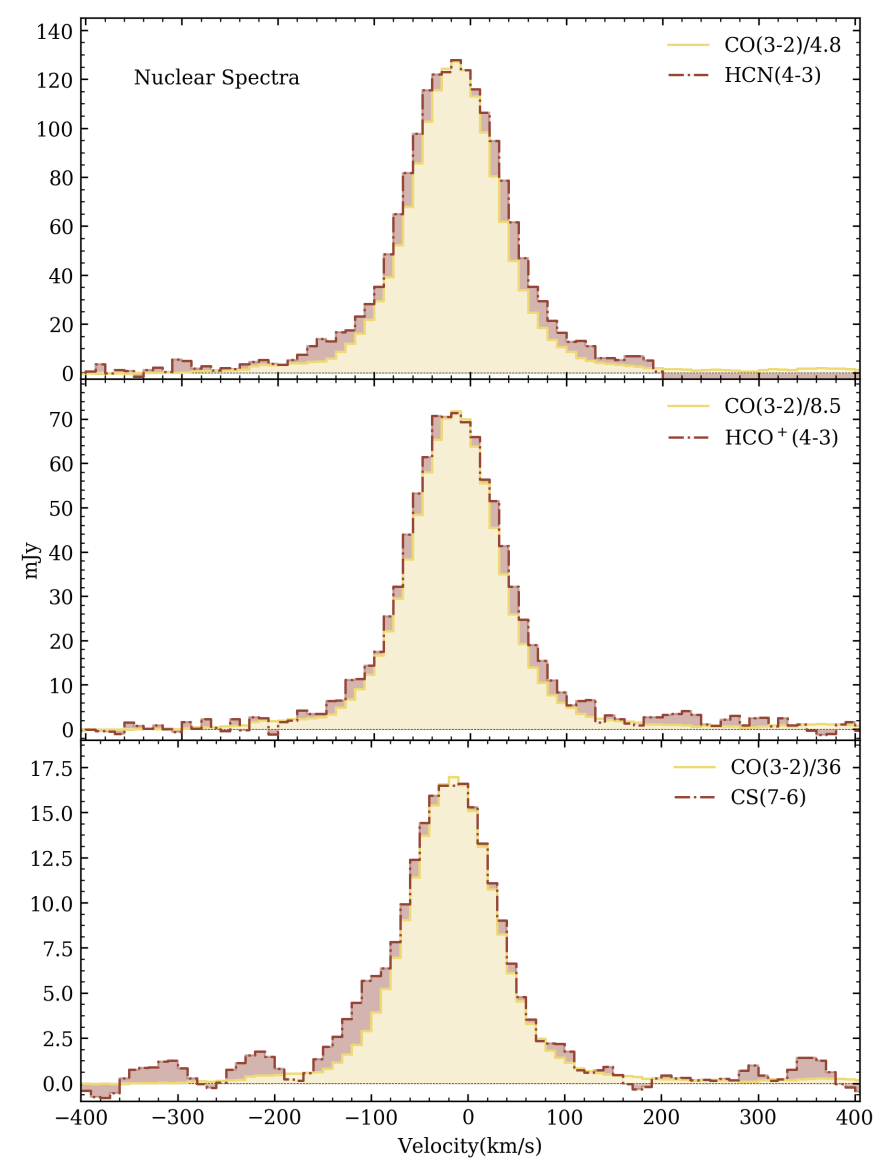

Fig. 17. Nuclear spectra extracted in an aperture of $r \sim 0.28^{\prime \prime}$ around the AGN position for $\mathrm{HCN}(4-3)$ (top), $\mathrm{HCO}^{+}(4-3)$ (middle), and CS(7-6) (bottom). The dense gas spectra were multiplied by a factor to scale with the $\mathrm{CO}(3-2)$ emission in order to compare their wing profiles.

\subsection{The nuclear molecular outflow in dense gas tracers}

We also detected the presence of broad wings in the nuclear spectrum of dense gas tracers $\mathrm{HCN}(4-3), \mathrm{HCO}^{+}(4-3)$, and CS(7-6), as indicated in Fig. 17. We also show the CO nuclear spectrum for comparison, and the high-velocity components cover the same velocity width of the CO wings $\left( \pm 300 \mathrm{~km} \mathrm{~s}^{-1}\right)$. We find that the ratio between the peak fluxes for the main core in the dense gas and $\mathrm{CO}$ are $\sim 5, \sim 8.5$, and $\sim 36$, for $\operatorname{HCN}(4-3)$, $\mathrm{HCO}^{+}(4-3)$, and $\mathrm{CS}(7-6)$, respectively. We also see some indication in Fig. 17 that, at least for $\operatorname{HCN}(4-3)$ and $\mathrm{CS}(7-6)$, the line ratios of the blue wings tend to be higher than the core.

In order to quantify the line ratio in the core and wings, we show the $\mathrm{HCN}(4-3) / \mathrm{CO}(3-2)$ along the nuclear spectra in Fig. 18. The ratio is shown up to velocities of $+200 \mathrm{~km} \mathrm{~s}^{-1}$ due to the tuning of Cycle 3 described in Sect. 2. The core component, defined by the disk rotation with velocities up to $\pm 100 \mathrm{~km} \mathrm{~s}^{-1}$, has a ratio of about 0.2 and the ratio increases for the high velocities towards the wings, up to values of $\sim 0.6$, suggesting an enhancement of HCN in the outflow. As discussed in Sect. 3.5, from analysis of the dense gas ratios in the submillimeter-HCN diagram (Izumi et al. 2016) in Fig. 12, the nuclear region of NGC 613 presents excitation conditions typical of XDRs in the vicinity of AGN. However, we find evidence that the $\mathrm{HCN}$ in the outflow can be three times higher than the values found in the nuclear CND.

A similar trend was also reported in the molecular outflow of the QSO galaxy Mrk231. The detection of the outflow in 


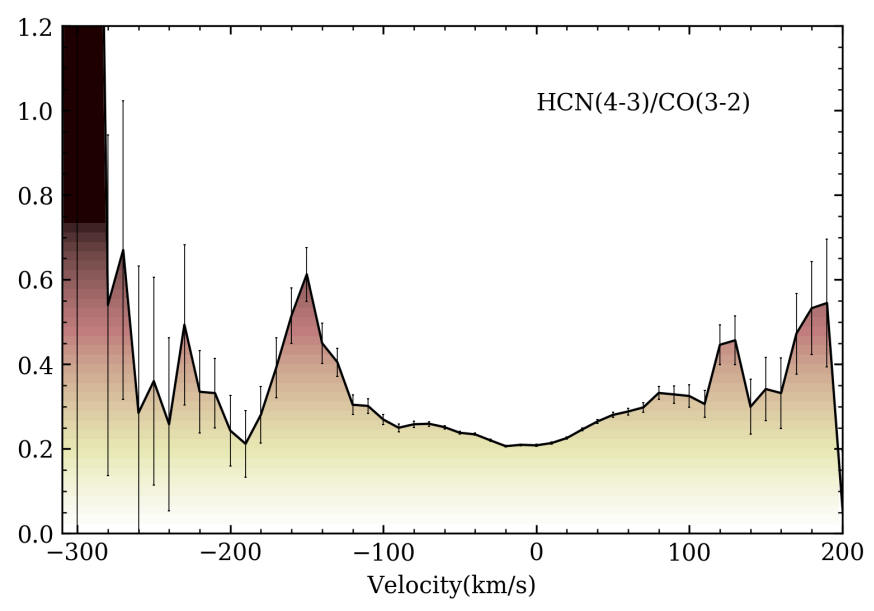

Fig. 18. $\mathrm{HCN}(4-3) / \mathrm{CO}(3-2)$ ratio in the nuclear spectra. The ratio is shown up to velocities of $+200 \mathrm{~km} \mathrm{~s}^{-1}$ due to the tuning of Cycle 3 described in Sect. 2. We can see that in the core, the ratio is $\sim 0.2$ and increases towards the wings up to values of $\sim 0.6$, indicating that the outflow is entrained mostly in a dense gas $\left(n \gtrsim 10^{4} \mathrm{~cm}^{-3}\right)$, as discussed in Aalto et al. (2012).

HCN(1-0) by Aalto et al. (2012) covers the same velocity range $\left( \pm 750 \mathrm{~km} \mathrm{~s}^{-1}\right)$ of the $\mathrm{CO}(1-0)$ outflow (Feruglio et al. 2010), and they found a high ratio of the $\mathrm{HCN} / \mathrm{CO} \sim 0.3-1$ in the outflow, higher than in the line core. The HCN is enhanced in the line wings by factors of between two and five, and they suggest that the outflow is mostly entrained in dense gas $n \gtrsim 10^{4} \mathrm{~cm}^{-3}$, which is consistent with the molecular gas being compressed and fragmented by shocks (Aalto et al. 2012). High-resolution observations of $\mathrm{HCN}$ and $\mathrm{HCO}^{+}$in the higher $J=3 \rightarrow 2$ transition exhibit prominent, spatially extended line wings for $\mathrm{HCN}(3-2)$ in Mrk 231 (Aalto et al. 2015). In Mrk 231 there were no line wings detected in $\mathrm{HCO}^{+}(3-2)$, while in $\mathrm{NGC} \mathrm{613,} \mathrm{there} \mathrm{is} \mathrm{some}$ indication of high-velocity gas. The $\mathrm{HCN}(4-3) / \mathrm{HCO}^{+}(4-3)$ ratios in the core are approximately equal to two, while in the wings can be two to three times higher, similar to what is found in Mrk 231 using the $\mathrm{HCN}(1-0) / \mathrm{HCO}+(1-0)$ ratios (Lindberg et al. 2016). Aalto et al. (2015) claimed that the elevated HCN abundance in the outflow is possibly caused by high temperatures in the X-ray-irradiated gas regions surrounding AGN (Harada et al. 2013).

Another possibility to explain the $\mathrm{HCN}$ enhancement in the outflow of NGC 613, is that the HCN emission stems from shocks potentially originating from the interaction of the ouflowing gas with the radio jet. The fact that the molecular outflow is spatially aligned with the central blob of the radio jet detected by Hummel \& Jorsater (1992) (see Fig. 16), a region where there is evidence of shock excitation as discussed in Sect. 3.6, corroborates this scenario. A further detailed analysis of the line ratios for the whole NUGA sample will be discussed in a future paper.

\subsection{Driving mechanism: AGN or star formation?}

The origin of the outflow might be related to star formation, which is concentrated in the nuclear ring region. The SFR can be estimated from the IR luminosity and the calibration from Kennicutt (1998b). From the IRAS fluxes, the IR luminosity is $L_{I R}=3 \times 10^{9} L_{\odot}$ (Table 1, Sturm et al. 2002), and the total SFR is equal to $5.3 M_{\odot} \mathrm{yr}^{-1}$. Based on the $\mathrm{H} \alpha$ luminosity associated with star formation in the central $3 \times 3 \mathrm{kpc}$ measured by
Davies et al. (2017), $L(\mathrm{H} \alpha)_{\mathrm{SF}}=5.28 \times 10^{41} \mathrm{erg} \mathrm{s}^{-1}$, we can also deduce, from Kennicutt's calibration, $\mathrm{SFR}=4.1 M_{\odot} \mathrm{yr}^{-1}$, which is consistent with the estimate from IR luminosity. If we isolate the contribution only coming from the circumnuclear starforming ring, the SFR estimated is SFR $=2.2 M_{\odot} \mathrm{yr}^{-1}$ in the ring of $r \lesssim 400 \mathrm{pc}$ (Mazzuca et al. 2008). Evidence of young starforming regions in the "hotspots" along the ring has recently been found by Falcón-Barroso et al. (2014). However, the value reported for NGC 613 in the nuclear region of $r \sim 40 \mathrm{pc}$ in aperture is very low: $\mathrm{SFR} \sim 0.015 M_{\odot} \mathrm{yr}$ (Falcón-Barroso et al. 2014). This low value could be due to extinction factors or a possible AGN contribution. The same nuclear region has a large reservoir of warm molecular gas (e.g., see Fig. 13), also found in other Seyfert galaxies (Hicks et al. 2013). Falcón-Barroso et al. (2014) suggest a cyclical episode of starburst about $\sim 10 \mathrm{Myr}$ ago, followed by another episode of nuclear activity.

We estimate a mass rate of $\dot{\mathrm{M}}_{\text {out }} \sim 27 M_{\odot} \mathrm{yr}^{-1}$ for the nuclear molecular outflow. Although this estimate is uncertain by a factor of a few given the unknown projection and the assumptions previously discussed in the text, the SFR in the nuclear region is about three orders of magnitude lower than $\dot{M}_{\text {out }}$. In general, galactic winds driven by starbursts correspond to mass-outflow rates of the same order as the SFR (e.g., Veilleux et al. 2005). Given the discrepancy between the SFR in the nuclear region and the mass-load rate of the outflow, we conclude that star formation alone is not able to drive the nuclear molecular outflow in NGC 613.

It has already been established that the mass-outflow rate increases with the AGN luminosity, supporting the idea of a luminous AGN pushing away the surrounding gas through a fast wind. Previous observational studies (Cicone et al. 2014; Carniani et al. 2015; Fiore et al. 2017) have shown that the molecular outflow properties are correlated with AGN luminosity, where the outflow kinetic power corresponds to about $5 \% L_{\mathrm{AGN}}$ and the momentum rate is $\sim 20 L_{\mathrm{AGN}} / c$, in agreement with theoretical models of AGN feedback (Faucher-Giguère \& Quataert 2012; Zubovas \& King 2012). For a sample of molecular and ionized outflows, Carniani et al. (2015) found that the ionized gas only traces a small fraction of the total gas mass, suggesting that the molecular phase dominates the outflow mass. This trend is also found by Fiore et al. (2017), but the ratio between molecular and ionized mass-outflow rates is reduced at the highest AGN bolometric luminosities. Nevertheless, these latter authors analyzed different samples of galaxies, and this conclusion could be affected by selection bias.

From XMM-Newton observations of NGC 613, Castangia et al. (2013) reported an X-ray luminosity of $\log L_{X}(2-10 \mathrm{keV})=$ $41.3 \mathrm{erg} \mathrm{s}^{-1}$. Applying a bolometric correction from Marconi et al. (2004) gives an AGN bolometric luminosity of $L_{\mathrm{AGN}, \mathrm{X}}=1.7 \times$ $10^{42} \mathrm{erg} \mathrm{s}^{-1}$. The bolometric luminosity derived by Davies et al. (2017) using the [OIII] emission associated only with the AGN contribution, traced as an extended ionization cone aligned with the radio jet, is $L_{\mathrm{AGN},[\mathrm{OIII}]}=4 \times 10^{42} \mathrm{erg} \mathrm{s}^{-1}$. If we include the shock and star forming contributions of the total $\mathrm{O}[\mathrm{III}]$ emission, we obtain $L_{\text {bol,[OIII] }}=3.75 \times 10^{43} \mathrm{erg} \mathrm{s}^{-1}$. The shock contribution most likely arises from the radio jet launched by the AGN, but here we cannot disentangle the contribution from star formation; the latter probably overestimates the bolometric AGN luminosity, while the former probably sets a lower limit.

In a recent study, Fluetsch et al. (2019) identified 45 molecular outflows in the local Universe using previous results from the literature and new detections from ALMA archive. These latter authors propose an even tighter empirical relation between the mass-outflow rate and the SFR, stellar mass, $M_{*}$, and the 
bolometric $L_{\mathrm{AGN}}$ :

$$
\begin{aligned}
\log \left(\dot{M}_{\text {out }}\right)= & 1.14 \log \left(0.52 \frac{\mathrm{SFR}}{M_{\odot} \mathrm{yr}^{-1}}+0.51 \frac{L_{\mathrm{AGN}}}{10^{43} \mathrm{erg} \mathrm{s}^{-1}}\right) \\
& -0.41 \log \left(\frac{M_{*}}{10^{11} M_{\odot}}\right),
\end{aligned}
$$

where the SFR is calculated from the total IR luminosity and $\dot{M}_{\text {out }}$ is in $M_{\odot} \mathrm{yr}^{-1}$. We adopt the bolometric AGN luminosity derived from the $\mathrm{O}[\mathrm{III}]$ emission line, $L_{\mathrm{AGN}}=4 \times 10^{42} \mathrm{erg} \mathrm{s}^{-1}$ (Davies et al. 2017), the total SFR inferred from the IRAS fluxes, $\mathrm{SFR}=5.3 M_{\odot} \mathrm{yr}^{-1}$, and the stellar mass of $M_{*}=4.5 \times 10^{10} M_{\odot}$ calculated in Paper I, derived from the $S^{4} \mathrm{G} 3.6 \mu \mathrm{m}$ IR image and the GALFIT decomposition (Salo et al. 2015). Hence, according to Eq. (5), we should expect an mass-outflow rate of $4.8 M_{\odot} \mathrm{yr}^{-1}$ in NGC 613, which is six times lower than our estimate.

The kinetic power of the nuclear outflow can be estimated as $P_{K \text {,out }}=0.5 v^{2} \dot{M}_{\text {out }}$. For the $\alpha_{\mathrm{CO}, \mathrm{MW}}$ assumption, $\dot{\mathrm{M}}_{\text {out }} \sim 27 M_{\odot} \mathrm{yr}^{-1}$, and we find $P_{K, \text { out }}=8 \times 10^{41} \mathrm{erg} \mathrm{s}^{-1}$, which corresponds to $20 \% L_{\mathrm{AGN}}$. This value exceeds the predictions from AGN feedback models and cosmological simulations that require that a fraction of the radiated luminosity be coupled to the surrounding gas $\sim 5 \% L_{\mathrm{AGN}}$ (Di Matteo et al. 2005; Zubovas \& King 2012). However, these predictions are based on powerful AGN accreting close to the Eddington limit, and we hypothesize that the coupling efficiency between AGNdriven outflows and $L_{\mathrm{AGN}}$ should be outweighed in LLAGN. The Eddington luminosity derived from the $\mathrm{BH}$ mass $\left(M_{\mathrm{BH}}=\right.$ $3.7 \times 10^{7} M_{\odot}$, Paper I) is $L_{\mathrm{Edd}}=4.6 \times 10^{45} \mathrm{erg} \mathrm{s}^{-1}$, leading to a low accretion rate $L_{\mathrm{AGN}} / L_{\mathrm{Edd}} \lesssim 1 \times 10^{-3}$. If we assume the lower $\alpha_{\mathrm{CO}}=1 / 4 \alpha_{\mathrm{CO}, \mathrm{MW}}$, the kinetic power of the outflow is $P_{K, \text { out }} \sim 2 \times 10^{41} \mathrm{erg} \mathrm{s}^{-1}$, and the coupling is $5 \% L_{\mathrm{AGN}}$. In both assumptions, the results indicate that the AGN can power the nuclear outflow in NGC 613, but the former requires a higher coupling efficiency.

The momentum flux of the outflow can be computed from $P_{\text {out }}=\dot{M}_{\text {out }} v \sim 5 \times 10^{34}$ dynes. Compared to the momentum provided by the AGN photons, $L_{\mathrm{AGN}} / c=1.3 \times 10^{32}$ dynes, it is higher by a factor of $\dot{M}_{\text {out }} v \sim 400 L_{\mathrm{AGN}} / c$. In case of energyconserved winds, AGN feedback models predict a momentum boost by factors up to 50 (e.g., Faucher-Giguère \& Quataert 2012). Even assuming the lower $\alpha_{\mathrm{O}}$, the value would exceed the predictions $\left(\sim 100 L_{\mathrm{AGN}} / c\right)$. One possibility to explain the high apparent energetics of the outflow in NGC 613 is that the AGN activity was stronger in the past. As discussed by Fluetsch et al. (2019), these authors found that $10 \%$ of the galaxies in their sample present outflows that exceed the theoretical predictions. They suggest these galaxies could have fossil outflows, resulting from a strong past AGN that has now faded. In the case of the LLAGN NGC 1377, a collimated molecular outflow is detected at $150 \mathrm{pc}$ scales (Aalto et al. 2016), possibly entrained by a faint radio jet, and these latter authors suggest that the nuclear activity of NGC 1377 may also be fading.

Alternatively, it is possible that the outflow is driven by the AGN radio jets. The radio power of the jet can be estimated from the $1.4 \mathrm{GHz}$ luminosity, $P_{1.4}$, using the relation of Bîrzan et al. (2008). If we compute the $P_{1.4}$ luminosity using the NRAO VLA Sky Survey (NVSS) flux measurement of $179.6 \mathrm{mJy}$ at $v=1.4 \mathrm{GHz}$ (Condon et al. 1998), we find a radio power of $P_{\text {jet }}=1.2 \times 10^{43} \mathrm{erg} \mathrm{s}^{-1}$. Since this value is elevated, it might include emission from the circumnuclear star-forming ring. To avoid this contribution, we used only the flux of the linear component of the radio jet at $4.86 \mathrm{GHz}$ by Hummel \& Jorsater (1992). At $4.86 \mathrm{GHz}$, the flux associated to the jet is
$S_{v}=7.2 \mathrm{mJy}$, and using a spectral index of $\alpha=-0.8$ to derive the flux at $1.4 \mathrm{GHz}$, we find $P_{\text {jet }}=5.5 \times 10^{42} \mathrm{erg} \mathrm{s}^{-1}$. Hydrodynamical simulations of the interaction of the jet with a clumpy interstellar medium has shown that the jet is able to drive a flow efficiently as soon as the Eddington ratio of the jet $P_{\text {jet }} / L_{\mathrm{Edd}}$ is larger than $10^{-4}$ (Wagner et al. 2012). This condition is required for the jet-driven outflow velocity to exceed the velocity dispersion of the $M-\sigma$ relation but it also depends on cloud size in the ISM. In NGC 613, this ratio is about $1.2 \times 10^{-3}$, and the jet power is about one or two orders of magnitude higher than the kinetic luminosity of the outflow. We conclude that the jet is able to drive the molecular outflow, even with low coupling. The molecular outflow in NGC 613 is an intriguing case where a very powerful molecular outflow is detected in a LLAGN. The SFR is very weak in the nuclear region and therefore unable to drive the flow. Radio jets are found to play a role in the driving mechanism to accelerate the molecular gas in other LLAGN. This is the case for the LINER NGC 1266, as suggested by Alatalo et al. (2011), which shows the presence of a weak radio jet (Nyland et al. 2013), and NGC 6764 (Leon et al. 2007) and the Seyfert 2 NGC 1433 (Combes et al. 2013). The properties of the flow require a contribution from the AGN through the entrainment of its radio jets.

\section{Torques and AGN fueling}

In Paper I, we reported the ALMA observations of the $\mathrm{CO}(3-2)$ line for all the galaxies in the NUGA sample. In three cases, NGC 613, NGC 1566, and NGC 1808, the CO emission revealed a nuclear trailing spiral, as in the large-scale one. Inside the nuclear ring at the ILR of the bar, usually a leading spiral is expected, developing transiently and generating positive torques, which drive the inner gas onto the ring. However, when the gravitational impact of the black hole is significant, the spiral can then be trailing, and the torques negative, and the gas is inflowing to fuel the nucleus (Buta \& Combes 1996; Fukuda et al. 1998).

We find filamentary structures in NGC 613, pointing to radial gas transport from the circumnuclear star-forming ring into the core region dominated by the AGN, as displayed in Fig. 4. The ring morphology appears disturbed by a radial outflow of material from the AGN, which is confirmed by the existence of a weak jet in archival radio maps. However, the radio jet does not seem to have any significant effect on the morphology of the large reservoir of molecular gas that has accumulated inside the central $100 \mathrm{pc}$.

If gas is inflowing from the bar dust lanes into the ring, as expected from gravity torques (García-Burillo et al. 2005), there is also an inflow in the CND, due to the nuclear trailing spiral, as already observed for NGC 1566 (Combes et al. 2014). Inside the nuclear spiral structure, there is a very dense and compact (radius $\sim 14 \mathrm{pc}$ ) rotating component, which might be interpreted as the molecular torus (Paper I).

In order to explore the efficiency of feeding in NGC 613, we estimated the gravitational torques exerted by the stellar potential on the molecular gas, following the methodology described by García-Burillo et al. (2005). The gravitational potential is computed in the plane of the galaxy using a red image (F814W) from HST, since 2MASS images have insufficient angular resolution. We did not separate the bulge from the disk contribution since NGC 613 is a late-type (Sbc) galaxy. This means that the bulge was effectively assumed to be flattened. Dark matter can be safely neglected inside the central kiloparsec. The image was rotated and deprojected according to $\mathrm{PA}=120^{\circ}$ and $i=41^{\circ}$, and then Fourier transformed to compute the gravitational potential 


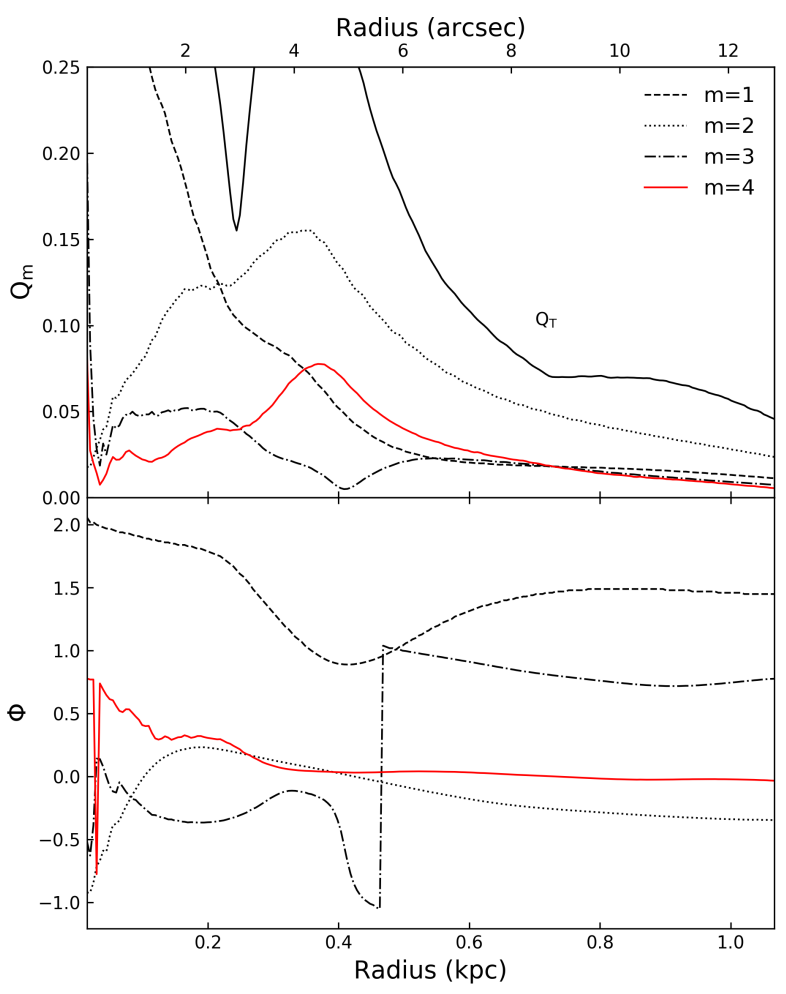

Fig. 19. Top: strengths $\left(Q_{m}\right.$ and total $\left.Q_{\mathrm{T}}\right)$ of the $m=1$ to $m=4$ Fourier components of the stellar potential within the central kiloparsec. The $m=1$ term is dominant up to a radius of $200 \mathrm{pc}$, and after the $m=2$ component dominates and has a constant phase, corresponding to the bar. Bottom: corresponding phases in radians of the Fourier components, taken from the major axis, in the deprojected image.

and forces. A stellar exponential disk thickness of approximately one twelfth of the radial scale-length of the galaxy $\left(h_{\mathrm{r}}=3.8 \mathrm{kpc}\right)$ was assumed, giving $h_{\mathrm{z}}=317 \mathrm{pc}$. This is the average scale ratio for galaxies of this type (e.g., Barnaby \& Thronson 1992; Bizyaev \& Mitronova 2009). The potential was obtained assuming a constant mass-to-light ratio of $M / L=0.5$ in the $I$-band over the considered portion of the image of $1 \mathrm{kpc}$ in size. This value is realistic in view of what is found statistically for spiral galaxies (Bell \& de Jong 2001). The pixel size of the map is $0.06^{\prime \prime}=5 \mathrm{pc}$, the average value between the ALMA and HST resolutions. The stellar M/L value was fit to reproduce the observed $\mathrm{CO}$ rotation curve.

The potential $\Phi(R, \theta)$ can be decomposed into its different Fourier components:

$\Phi(R, \theta)=\Phi_{0}(R)+\sum_{m} \Phi_{m}(R) \cos \left(m \theta-\phi_{m}(R)\right)$,

where $\Phi_{m}(R)$ and $\phi_{m}(R)$ are the amplitude and the phase of the $m$-mode, respectively. The strength of the $m$-Fourier component, $Q_{m}(R)$, is defined as $Q_{m}(R)=m \Phi_{m} / R\left|F_{0}(R)\right|$, that is, by the ratio between tangential and radial forces (Combes \& Sanders 1981). The strength of the total nonaxisymmetric perturbation $Q_{T}(R)$ is defined similarly with the maximum amplitude of the tangential force $F_{T}^{\max }(R)$. Their radial distributions and the radial phase variations are displayed in Fig. 19.

The derivatives of the potential yield the forces per unit mass $\left(F_{x}\right.$ and $\left.F_{y}\right)$ at each pixel, and the torques per unit mass $t(x, y)$ are then computed by $t(x, y)=x F_{y}-y F_{x}$. The sign of the torque is determined relative to the sense of rotation in the plane of the galaxy. The product of the torque and the gas density $\Sigma$ at each

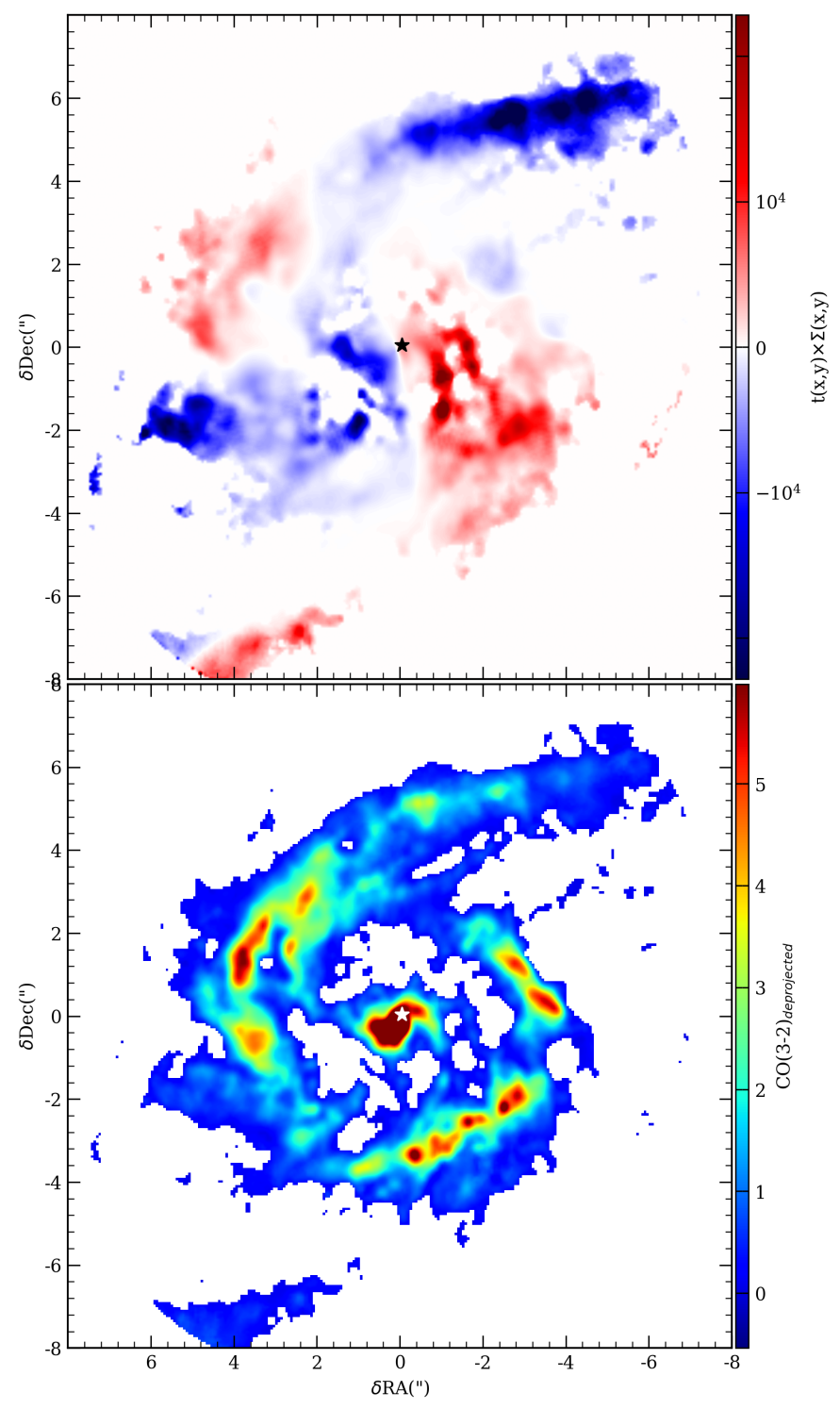

Fig. 20. Top: map of the gravitational torque, $(t(x, y) \times \Sigma(x, y)$, as defined in the text) in the center of NGC 613. The torques change sign as expected in a four-quadrant pattern (or butterfly diagram). The orientation of the quadrants follows the nuclear bar's orientation. In this deprojected picture, the major axis of the galaxy is oriented parallel to the horizontal axis. Bottom: deprojected image of the $\mathrm{CO}(3-2)$ emission at the same scale and with the same orientation, for comparison. The color scales are linear, in arbitrary units.

pixel allows one to then derive the net effect on the gas at each radius. This quantity $t(x, y) \times \Sigma(x, y)$, is shown in Fig. 20, together with the deprojected $\mathrm{CO}$ map.

The torque weighted by the gas density $\Sigma(x, y)$ is then averaged over azimuth, that is,

$t(R)=\frac{\int_{\theta} \Sigma(x, y) \times\left(x F_{y}-y F_{x}\right)}{\int_{\theta} \Sigma(x, y)}$.

The quantity $t(R)$ represents the time derivative of the specific angular momentum $L$ of the gas averaged azimuthally (GarcíaBurillo et al. 2005). Normalizing at each radius by the angular momentum and rotation period $\left(\mathrm{T}_{\text {rot }}\right)$ allows us to estimate the efficiency of the gas flow, as shown in Fig. 21. The torques are negative in the winding arms at $r \sim 500 \mathrm{pc}$ (corresponding to 


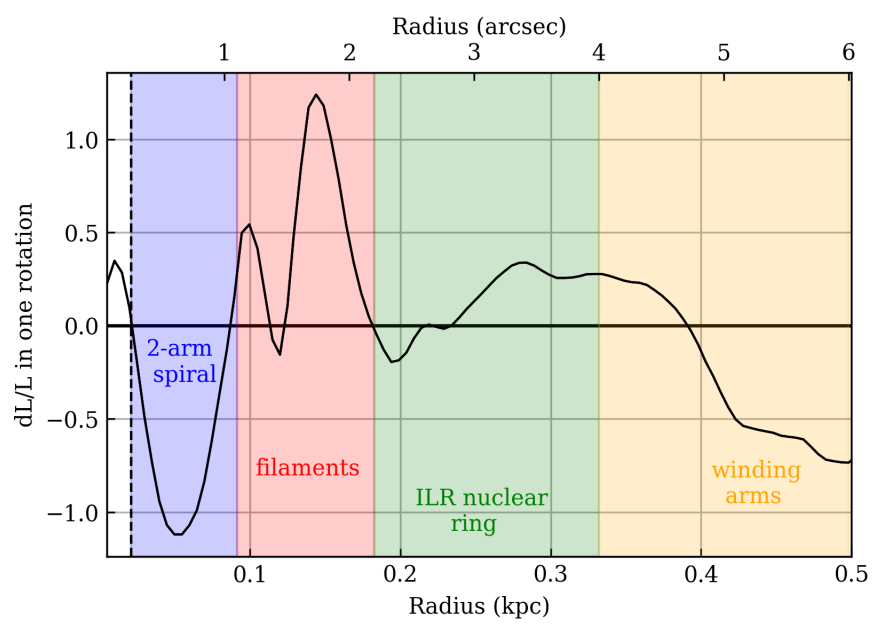

Fig. 21. Radial distribution of the torque, quantified by the fraction of the angular momentum transferred from the gas in one rotation- $\mathrm{d} L / L$, estimated from the $\mathrm{CO}(3-2)$ deprojected map. The vertical dashed line at $25 \mathrm{pc}$ radius delimitates the extent of the central gas outflow, and the computation has no meaning here. The torque is negative inside the $\lesssim 100$ pc nuclear spiral and in the winding arms and positive in the filaments.

the dust lanes) and are indeed contributing to drive the outer gas into the star-forming ring at $\sim 350 \mathrm{pc}$. The observations of nuclear rings are more common among barred galaxies (Kormendy \& Kennicutt 2004; Peeples \& Martini 2006; Jogee et al. 2006), and can be explained by the gas slowing down as it crosses the ILR, consequently weakening the gravitational torques, and the gas piles up in rings (Combes \& Gerin 1985; Byrd et al. 1994). We can see in Fig. 21 that the efficiency certainly drops in the inner ILR region. The filaments within the nuclear region, between 100 and $200 \mathrm{pc}$, show that the gas gains angular momentum in one rotation, which is $T_{\text {rot }} \sim 12 \mathrm{Myr}$.

The nuclear bar strength is moderate, however the $\mathrm{BH}$ has a strong influence on the nuclear molecular gas, as shown in Fig. 21, and the fueling efficiency is high in the nuclear spiral. Between 25 and $100 \mathrm{pc}$, the gas loses its angular momentum in one rotation, which is $T_{\text {rot }}(100 \mathrm{pc}) \sim 9.5 \mathrm{Myr}$. Inside $25 \mathrm{pc}$, the torques are positive and correspond to the region where we detect the molecular outflow. Since the gas in this region is not in quasi-stationary orbits, but is rather ejected from the galaxy plane, the computation cannot be interpreted in terms of the average torque here.

As shown in Fig. 20, the nuclear spiral structure inside the ILR ring of the bar is of a trailing nature and is located inside the negative torque quadrants. This might appear surprising, since in many cases the spiral structure is predicted to be leading in this region, and the torque positive, maintaining the gas in the ILR ring (Buta \& Combes 1996). However, in the presence of a sufficiently massive black hole, this behavior can be reversed: the spiral becomes trailing, and the torque negative. Indeed, the indicator of the precession rate of elliptical orbits in the frame of the epicyclic approximation, $\Omega-\kappa / 2$, is significantly modified by a central massive body. Instead of decreasing regularly towards zero at the center, the $\Omega-\kappa / 2$ curve increases steeply as $r^{-3 / 2}$. The gas undergoes collisions, loses energy and spirals progressively towards the center. When the precession rate decreases, the series of elliptical orbits precess more and more slowly, and then lag at smaller radii, forming a leading structure; when the precession rate increases, they form a trailing structure (Combes et al. 2014).

One way to detect spiral structure in the nuclear gas is to amplify the dust extinction features in the HST images.
However, we would like to stress that observations of spiral dust lanes interior to the star-formation rings are not very common (Martini et al. 2003; Peeples \& Martini 2006). Notwithstanding, a clear case is seen in NGC 2207, where dusty spirals extend from $\sim 50$ to $300 \mathrm{pc}$, suggesting that the gas continues to sink inside the ILR and might be promoting gas accretion onto the nucleus (Elmegreen et al. 1998). Multiple spiral arms were also detected inside the star-forming ring $(r \sim 700 \mathrm{pc})$ in the LINER/Sy 1 galaxy NGC 1097 by Prieto et al. (2005). These latter authors compared their results with hydrodynamic models of Maciejewski (2004), concluding that inflows were occurring along the nuclear spirals. Although nuclear star forming rings are common, their interior structure is difficult to distinguish in dust maps, making the molecular gas emission a better tracer. The NUGA maps show clear evidence of trailing spirals in three objects of the sample, highlighting the importance of the highresolution ALMA observations.

NGC 613 is therefore an example of a trailing spiral inside the nuclear ring of a bar; for example, see the case described in Buta \& Combes (1996). This means that the mass of the black hole should be sufficiently high to have an influence on the gas dynamics on a $100 \mathrm{pc}$ scale. In summary, the gravity torques are negative in the winding spirals, and the gas accumulates in the star forming ring at the inner ILR of the nuclear bar. The filamentary structure gains angular momentum and then the nuclear spiral at $\lesssim 100 \mathrm{pc}$ present a very high efficiency in fuelling the central $\mathrm{BH}$.

\section{Conclusions}

Here we present the combined ALMA cycle 3 and 4 observations for the Seyfert/nuclear starburst galaxy NGC 613. The combined observations in $\mathrm{CO}(3-2)$ reach a spatial resolution of $0.2^{\prime \prime} \sim 17 \mathrm{pc}$. We study the morphology and the kinematics of the molecular gas in the central $1 \mathrm{kpc}$ and our main findings are summarized below:

- The $350 \mathrm{GHz}$ continuum map shows a compact, barely resolved emission peak at the position of the AGN, surrounded by a patchy ring, matching the star-forming ring seen on optical images.

- The morphology of $\mathrm{CO}(3-2)$ line emission reveals several components: a two-arm nuclear spiral at $r \lesssim 100$ pc trailing the gas toward the center, and a circumnuclear ring at $\sim 350 \mathrm{pc}$ corresponding to the star-forming ring. Also, we find evidence of a filamentary structure connecting the ring and the nuclear spiral. The ring reveals two breaks into two winding spiral arms towards the NW and SE corresponding to the dust lanes in the HST images.

- The kinematics of the $\mathrm{CO}$ emission show a rather regular rotational velocity field in the inner kiloparsec disk. We applied a tilted-ring model to fit the velocity map and the residuals indeed show the bulk of the molecular gas in circular motion except in the west part of the ring, the perturbations of which are due to the contact point between the ring and the SE winding arm.

- There is a remarkable coincidence between the molecular gas and the warm $\mathrm{H}_{2}$ and ionized gas traced by the [FeII] and $\mathrm{Br} \gamma$ emission lines in the star-forming ring. Line diagnostics in the NIR indicate that the clumps in the ring are in agreement with young star-forming excitation. On the other hand, the nucleus of NGC 613 presents an excitation mechanism typical of Seyferts or LINERs.

- We measured the line intensity ratios $R_{\mathrm{HCN} / \mathrm{HCO}}^{+}$and $R_{\mathrm{HCN} / \mathrm{CS}}$ in the nuclear region and in a clump along the ring. We find that the ratio for the nuclear region points to the 
AGN-dominated part of the HCN-submillimeter diagram, while the ratio for the clump is located in the starburstdominated part. These results indicate that the nuclear region of NGC 613 presents line ratios in agreement with excitation conditions typical of XDRs in the vicinity of AGN.

- In the PV diagrams, we find skewed kinematics in the nuclear region of $r \sim 25 \mathrm{pc}$. This feature is seen as broad wings $\left(v \pm 300 \mathrm{~km} \mathrm{~s}^{-1}\right)$ in the $\mathrm{CO}$ nuclear spectrum, and the wings are also present in the dense gas tracers. We identify this feature as a molecular outflow emanating from the nucleus. The molecular outflow is co-spatial with the central blob detected in the radio jet.

- We derive the molecular mass associated to the outflow as $M_{\text {out }}=2 \times 10^{6} M_{\odot}$. The mass outflow rate is $\dot{M}_{\text {out }}=$ $27 M_{\odot} \mathrm{yr}^{-1}$. If instead, we use the mass derived assuming the typical values for galaxy centers, $\alpha_{\mathrm{CO}}=\frac{1}{4} \alpha_{\mathrm{CO}, \mathrm{MW}}$, we find that the mass load rate is $\dot{M}_{\text {out }} \sim 7 M_{\odot} \mathrm{yr}^{-1}$.

- We find a $\mathrm{HCN}$ enhancement in the outflow probed by an increase in the $\mathrm{HCN}(4-3) / \mathrm{CO}(3-2)$ ratio along the wings in the nuclear spectra. While the core has ratios of about $\sim 0.2$, this value increases in the wings up to $\sim 0.6$, indicating that the outflow is entrained mostly in a dense gas $\left(n \gtrsim 10^{4} \mathrm{~cm}^{-3}\right)$. Another possibility is that the $\mathrm{HCN}$ emission stems from shocks potentially originating from the interaction of the ouflowing gas with the radio jet.

- The molecular outflow energetics exceed the values predicted by AGN-feedback models. The kinetic power of the nuclear outflow corresponds to $P_{K \text {,out }}=20 \% L_{\mathrm{AGN}}$ and the momentum rate is $\dot{M}_{\text {out }} v \sim 400 L_{\mathrm{AGN} / c}$. We speculate that, given its current weak nuclear activity, NGC 613 might be a case of fossil outflows resulting from a strong AGN that has now faded.

- The outflow can be entrained by its radio jet. We find that $P_{\text {jet }} / L_{\text {Edd }} \sim 10^{-3}$ and the jet power is about one or two orders of magnitudes higher than the kinetic luminosity of the outflow. In these conditions, the jet is able to drive the molecular outflow.

- The trailing spiral observed in CO emission is inside the ILR ring of the bar. We computed the gravitational potential from the stars within the central kiloparsec from the $I$-band HST image. Weighting the torques on each pixel by the gas surface density observed in the $\mathrm{CO}(3-2)$ line allowed us to estimate the sense of the angular momentum exchange and its efficiency. The gravity torques are negative from 25 to $100 \mathrm{pc}$. Between 50 and $100 \mathrm{pc}$, the gas loses its angular momentum in a rotation period, providing evidence of fueling of the AGN.

The molecular outflow in NGC 613 is an intriguing case where a very powerful molecular outflow is detected in a LLAGN. The SFR is very weak in the nuclear region, and is therefore not able to drive the flow. The properties of the flow require the contribution of the AGN through the entrainment of its radio jets. On the other hand, there is a clear trailing spiral observed in molecular gas inside the ILR ring of a bar, indicating that the SMBH is influencing the gas dynamics. Instead of maintaining the ILR ring density, the torques are then driving gas towards the nucleus, a first step towards possibly fueling the AGN. NGC 613 is a remarkable example of the complexity of fuelling and feedback mechanisms in AGN, and reinforces the importance of detailed analysis of nearby galaxies with ALMA capabilities to shed light on the gas-flow cycle in AGN.

Acknowledgements. We warmly thank the referee for constructive comments and suggestions. The ALMA staff in Chile and ARC-people at IRAM are gratefully acknowledged for their help in the data reduction. We particularly thank Philippe Salomé for useful advice and Jesús Falcón-Barroso for the SINFONI and VLA data. AA would like to thank the Brazilian scholarship program Science without Borders - CNPq (reference [234043/2014-8]) for financial support. SGB acknowledges support from the Spanish MINECO grant AYA2016-76682-C3-2-P. LH is grateful to INAF PRIN-SKA funding program 1.05.01.88.04. AE thanks the Collaborative Research Centre 956, sub-project [A02], funded by the Deutsche Forschungsgemeinschaft (DFG) project ID 184018867. This paper makes use of the following ALMA data: ADS/JAO. ALMA\#2015.0.00404.S, and ADS/JAO.ALMA\#2016.0.00296.S. ALMA is a partnership of ESO (representing its member states), NSF (USA) and NINS (Japan), together with NRC (Canada) and NSC and ASIAA (Taiwan), in cooperation with the Republic of Chile. The Joint ALMA Observatory is operated by ESO, AUI/NRAO and NAOJ. The National Radio Astronomy Observatory is a facility of the National Science Foundation operated under cooperative agreement by Associated Universities, Inc. We used observations made with the NASA/ESA Hubble Space Telescope, and obtained from the Hubble Legacy Archive, which is a collaboration between the Space Telescope Science Institute (STScI/NASA), the Space Telescope European Coordinating Facility (ST-ECF/ESA), and the Canadian Astronomy Data Centre (CADC/NRC/CSA). We made use of the NASA/IPAC Extragalactic Database (NED), and of the HyperLeda database. This research made use of Astropy, a community developed core Python package for Astronomy. This work was supported by the Programme National Cosmology et Galaxies (PNCG) of CNRS/INSU with INP and IN2P3, co-funded by CEA and CNES.

\section{References}

Aalto, S., Garcia-Burillo, S., Muller, S., et al. 2012, A\&A, 537, A44 Aalto, S., Garcia-Burillo, S., Muller, S., et al. 2015, A\&A, 574, A85 Aalto, S., Costagliola, F., Muller, S., et al. 2016, A\&A, 590, A73

Alatalo, K., Blitz, L., Young, L. M., et al. 2011, ApJ, 735, 88

Alonso-Herrero, A., Rieke, M. J., Rieke, G. H., \& Ruiz, M. 1997, ApJ, 482, 747 Antonucci, R. 1993, ARA\&A, 31, 473

Bajaja, E., Wielebinski, R., Reuter, H.-P., Harnett, J. I., \& Hummel, E. 1995, A\&AS, 114, 147

Baldwin, J. A., Phillips, M. M., \& Terlevich, R. 1981, PASP, 93, 5

Barcos-Muñoz, L., Aalto, S., Thompson, T. A., et al. 2018, ApJ, 853, L28

Barnaby, D., Thronson, Jr., H. A., et al. 1992, AJ, 103, 41

Bell, E. F., \& de Jong, R. S. 2001, ApJ, 550, 212

Bertola, F., Bettoni, D., Danziger, J., et al. 1991, ApJ, 373, 369

Bîrzan, L., McNamara, B. R., Nulsen, P. E. J., Carilli, C. L., \& Wise, M. W. 2008,

ApJ, 686, 859

Bizyaev, D., \& Mitronova, S. 2009, ApJ, 702, 1567

Blackman, C. P. 1981, MNRAS, 195, 451

Böker, T., Falcón-Barroso, J., Schinnerer, E., Knapen, J. H., \& Ryder, S. 2008, AJ, 135, 479

Bolatto, A. D., Wolfire, M., \& Leroy, A. K. 2013, ARA\&A, 51, 207

Burbidge, E. M., Burbidge, G. R., Rubin, V. C., \& Prendergast, K. H. 1964, ApJ, 140,85

Buta, R., \& Combes, F. 1996, Fund. Cosmic Phys., 17, 95

Byrd, G., Rautiainen, P., Salo, H., Buta, R., \& Crocher, D. A. 1994, AJ, 108, 476 Carniani, S., Marconi, A., Maiolino, R., et al. 2015, A\&A, 580, A102

Castangia, P., Panessa, F., Henkel, C., Kadler, M., \& Tarchi, A. 2013, MNRAS, 436, 3388

Cicone, C., Maiolino, R., Sturm, E., et al. 2014, A\&A, 562, A21

Colina, L., Piqueras López, J., Arribas, S., et al. 2015, A\&A, 578, A48

Combes, F. 2003, in Active Galactic Nuclei: From Central Engine to Host

Galaxy, eds. S. Collin, \& F. Combes, ASP Conf. Ser., 290, 411

Combes, F., \& Gerin, M. 1985, A\&A, 150, 327

Combes, F., \& Sanders, R. H. 1981, A\&A, 96, 164

Combes, F., García-Burillo, S., Casasola, V., et al. 2013, A\&A, 558, A124

Combes, F., García-Burillo, S., Casasola, V., et al. 2014, A\&A, 565, A97

Combes, F., García-Burillo, S., Audibert, A., et al. 2019, A\&A, 623, A79

Comerón, S., Knapen, J. H., Beckman, J. E., et al. 2010, MNRAS, 402, 2462

Condon, J. J., Cotton, W. D., Greisen, E. W., et al. 1998, AJ, 115, 1693

Croton, D. J., Springel, V., White, S. D. M., et al. 2006, MNRAS, 365, 11

Dame, T. M., Hartmann, D., \& Thaddeus, P. 2001, ApJ, 547, 792

Dasyra, K. M., \& Combes, F. 2012, A\&A, 541, L7

Dasyra, K. M., Combes, F., Novak, G. S., et al. 2014, A\&A, 565, A46

Dasyra, K. M., Combes, F., Oosterloo, T., et al. 2016, A\&A, 595, L7

Davies, R. L., Groves, B., Kewley, L. J., et al. 2017, MNRAS, 470, 4974

Davis, B. L., Berrier, J. C., Johns, L., et al. 2014, ApJ, 789, 124

de Vaucouleurs, G., de Vaucouleurs, A., Corwin, Jr., H. G., et al. 1991, Third Reference Catalogue of Bright Galaxies. Volume I: Explanations and references. Volume II: Data for galaxies between $0 \mathrm{~h}$ and $12 \mathrm{~h}$. Volume III: Data for galaxies between $12 \mathrm{~h}$ and $24 \mathrm{~h}$.

Di Matteo, T., Springel, V., \& Hernquist, L. 2005, Nature, 433, 604 
Di Matteo, T., Colberg, J., Springel, V., Hernquist, L., \& Sijacki, D. 2008, ApJ, 676, 33

Di Teodoro, E. M., \& Fraternali, F. 2015, MNRAS, 451, 3021

Elmegreen, B. G., Elmegreen, D. M., Brinks, E., et al. 1998, ApJ, 503, L119

Falcón-Barroso, J., Ramos Almeida, C., Böker, T., et al. 2014, MNRAS, 438, 329

Faucher-Giguère, C.-A., \& Quataert, E. 2012, MNRAS, 425, 605

Feruglio, C., Maiolino, R., Piconcelli, E., et al. 2010, A\&A, 518, L155

Feruglio, C., Ferrara, A., Bischetti, M., et al. 2017, A\&A, 608, A30

Fiore, F., Feruglio, C., Shankar, F., et al. 2017, A\&A, 601, A143

Fischer, J., Sturm, E., González-Alfonso, E., et al. 2010, A\&A, 518, L41

Fluetsch, A., Maiolino, R., Carniani, S., et al. 2019, MNRAS, 483, 4586

Forbes, D. A., \& Ward, M. J. 1993, ApJ, 416, 150

Fukuda, H., Wada, K., \& Habe, A. 1998, MNRAS, 295, 463

Gadotti, D. A., Sánchez-Blázquez, P., Falcón-Barroso, J., et al. 2019, MNRAS, 482, 506

Gallimore, J. F., Elitzur, M., Maiolino, R., et al. 2016, ApJ, 829, L7

García-Burillo, S. 2016, in From Interstellar Clouds to Star-Forming Galaxies Universal Processes?, eds. P. Jablonka, P. André, \& F. van der Tak, IAU Symp., 315, 207

García-Burillo, S., \& Combes, F. 2012, J. Phys. Conf. Ser., 372, 012050

García-Burillo, S., Combes, F., Schinnerer, E., Boone, F., \& Hunt, L. K. 2005 A\&A, 441, 1011

García-Burillo, S., Combes, F., Usero, A., et al. 2014, A\&A, 567, A125

García-Burillo, S., Combes, F., Ramos Almeida, C., et al. 2016, ApJ, 823, L12

Ginsburg, A., \& Mirocha, J. 2011, Astrophysics Source Code Library [record ascl:1109.001]

Ginsburg, A., Robitaille, T., Beaumont, C., et al. 2015, in Revolution in Astronomy with ALMA: The Third Year, eds. D. Iono, K. Tatematsu, A. Wootten, L. Testi, et al., ASP Conf. Ser., 499, 363

Goulding, A. D., \& Alexander, D. M. 2009, MNRAS, 398, 1165

Guilloteau, S., \& Lucas, R. 2000, in Imaging at Radio through Submillimeter Wavelengths, eds. J. G. Mangum, \& S. J. E. Radford, ASP Conf. Ser., 217, 299

Gültekin, K., Richstone, D. O., Gebhardt, K., et al. 2009, ApJ, 698, 198

Harada, N., Thompson, T. A., \& Herbst, E. 2013, ApJ, 765, 108

Hicks, E. K. S., Davies, R. I., Maciejewski, W., et al. 2013, ApJ, 768, 107

Ho, L. C., \& Kim, M. 2014, ApJ, 789, 17

Ho, L. C., Li, Z.-Y., Barth, A. J., Seigar, M. S., \& Peng, C. Y. 2011, ApJS, 197, 21

Hopkins, P. F., \& Quataert, E. 2010, MNRAS, 407, 1529

Hopkins, P. F., Hernquist, L., Cox, T. J., et al. 2006, ApJS, 163, 1

Hopkins, P. F., Hayward, C. C., Narayanan, D., \& Hernquist, L. 2012, MNRAS 420,320

Hummel, E., \& Jorsater, S. 1992, A\&A, 261, 85

Hummel, E., Jorsater, S., Lindblad, P. O., \& Sandqvist, A. 1987, A\&A, 172, 51

Hunt, L. K., Combes, F., García-Burillo, S., et al. 2008, A\&A, 482, 133

Imanishi, M., Nakanishi, K., \& Izumi, T. 2016, AJ, 152, 218

Imanishi, M., Nakanishi, K., \& Izumi, T. 2018, ApJ, 856, 143

Israel, F. P. 2009a, A\&A, 506, 689

Israel, F. P. 2009b, A\&A, 493, 525

Izumi, T., Kohno, K., Aalto, S., et al. 2016, ApJ, 818, 42

Jogee, S. 2006, in Physics of Active Galactic Nuclei at all Scales, ed. D. Alloin

(Berlin: Springer Verlag), Lect. Notes Phys., 693, 143

Jungwiert, B., Combes, F., \& Axon, D. J. 1997, A\&AS, 125, 479

Kennicutt, Jr., R. C. 1998a, ApJ, 498, 541

Kennicutt, Jr., R. C. 1998b, ARA\&A, 36, 189

Kohno, K. 2005, in The Evolution of Starbursts, eds. S. Hüttmeister, E. Manthey, D. Bomans, \& K. Weis, Am. Inst. Phys. Conf. Ser., 783, 203

Kondratko, P. T., Greenhill, L. J., Moran, J. M., et al. 2006, ApJ, 638, 100

Kormendy, J., \& Kennicutt, Jr., R. C. 2004, ARA\&A, 42, 603

Krips, M., Neri, R., García-Burillo, S., et al. 2008, ApJ, 677, 262
Krips, M., Martín, S., Eckart, A., et al. 2011, ApJ, 736, 37 Leon, S., Eckart, A., Laine, S., et al. 2007, A\&A, 473, 747 Li, Z.-Y., Ho, L. C., Barth, A. J., \& Peng, C. Y. 2011, ApJS, 197, 22 Lindberg, J. E., Aalto, S., Muller, S., et al. 2016, A\&A, 587, A15

Liu, J.-F., \& Bregman, J. N. 2005, ApJS, 157, 59

Maciejewski, W. 2004, MNRAS, 354, 892

Magorrian, J., Tremaine, S., Richstone, D., et al. 1998, AJ, 115, 2285

Mao, R.-Q., Schulz, A., Henkel, C., et al. 2010, ApJ, 724, 1336

Marconi, A., Risaliti, G., Gilli, R., et al. 2004, MNRAS, 351, 169

Martini, P., Regan, M. W., Mulchaey, J. S., \& Pogge, R. W. 2003, ApJ, 589, 774

Matsushita, S., Muller, S., \& Lim, J. 2007, A\&A, 468, L49

Mazzalay, X., Saglia, R. P., Erwin, P., et al. 2013, MNRAS, 428, 2389

Mazzuca, L. M., Knapen, J. H., Veilleux, S., \& Regan, M. W. 2008, ApJS, 174, 337

McConnell, N. J., \& Ma, C.-P. 2013, ApJ, 764, 184

McMullin, J. P., Waters, B., Schiebel, D., Young, W., \& Golap, K. 2007, in Astronomical Data Analysis Software and Systems XVI, eds. R. A. Shaw, F. Hill, \& D. J. Bell, ASP Conf. Ser., 376, 127

Miyamoto, Y., Nakai, N., Seta, M., et al. 2017, PASJ, 69, 83

Nyland, K., Alatalo, K., Wrobel, J. M., et al. 2013, ApJ, 779, 173

Onodera, S., Kuno, N., Tosaki, T., et al. 2010, ApJ, 722, L127

Peeples, M. S., \& Martini, P. 2006, ApJ, 652, 1097

Piner, B. G., Stone, J. M., \& Teuben, P. J. 1995, ApJ, 449, 508

Prieto, M. A., Maciejewski, W., \& Reunanen, J. 2005, AJ, 130, 1472

Querejeta, M., Schinnerer, E., García-Burillo, S., et al. 2016, A\&A, 593, A118

Regan, M. W., \& Teuben, P. J. 2004, ApJ, 600, 595

Riffel, R. A., \& Storchi-Bergmann, T. 2011, MNRAS, 411, 469

Riffel, R., Rodríguez-Ardila, A., Aleman, I., et al. 2013, MNRAS, 430, 2002

Robitaille, T., \& Bressert, E. 2012, Astrophysics Source Code Library [record ascl:1208.017]

Rodríguez-Ardila, A., Pastoriza, M. G., Viegas, S., Sigut, T. A. A., \& Pradhan, A. K. 2004, A\&A, 425, 457

Rogstad, D. H., Lockhart, I. A., \& Wright, M. C. H. 1974, ApJ, 193, 309

Rupke, D. S., Veilleux, S., \& Sanders, D. B. 2005a, ApJ, 632, 751

Rupke, D. S., Veilleux, S., \& Sanders, D. B. 2005b, ApJS, 160, 115

Sakamoto, K., Aalto, S., Combes, F., Evans, A., \& Peck, A. 2014, ApJ, 797, 90

Salo, H., Laurikainen, E., Laine, J., et al. 2015, ApJS, 219, 4

Sandstrom, K. M., Leroy, A. K., Walter, F., et al. 2013, ApJ, 777, 5

Schinnerer, E., Eckart, A., Tacconi, L. J., Genzel, R., \& Downes, D. 2000, ApJ, 533,850

Schmidt, M. 1959, ApJ, 129, 243

Seigar, M. S., Harrington, A., \& Treuthardt, P. 2018, MNRAS, 481, 5394

Shlosman, I., Frank, J., \& Begelman, M. C. 1989, Nature, 338, 45

Sijacki, D., Springel, V., Di Matteo, T., \& Hernquist, L. 2007, MNRAS, 380, 877

Solomon, P. M., \& Vanden Bout, P. A. 2005, ARA\&A, 43, 677

Steer, I., Madore, B. F., Mazzarella, J. M., et al. 2017, AJ, 153, 37

Sturm, E., Lutz, D., Verma, A., et al. 2002, A\&A, 393, 821

Sturm, E., González-Alfonso, E., Veilleux, S., et al. 2011, ApJ, 733, L16

Tacconi, L. J., Neri, R., Genzel, R., et al. 2013, ApJ, 768, 74

Usero, A., García-Burillo, S., Fuente, A., Martín-Pintado, J., \& RodríguezFernández, N. J. 2004, A\&A, 419, 897

van den Bosch, R. C. E. 2016, ApJ, 831, 134

Veilleux, S., Cecil, G., \& Bland-Hawthorn, J. 2005, ARA\&A, 43, 769

Veilleux, S., Meléndez, M., Sturm, E., et al. 2013, ApJ, 776, 27

Veilleux, S., Bolatto, A., Tombesi, F., et al. 2017, ApJ, 843, 18

Veron-Cetty, M.-P., \& Veron, P. 1986, A\&AS, 66, 335

Viti, S., García-Burillo, S., Fuente, A., et al. 2014, A\&A, 570, A28

Wada, K. 2004, Coevolution of Black Holes and Galaxies, 186

Wagner, A. Y., Bicknell, G. V., \& Umemura, M. 2012, ApJ, 757, 136

Zubovas, K., \& King, A. 2012, ApJ, 745, L34

Zubovas, K., \& King, A. R. 2014, MNRAS, 439, 400 